\title{
6G VISION: AN ULTRA-FLEXIBLE PERSPECTIVE
}

\author{
Ahmet Yazar ${ }^{1}$, Seda Doğan Tusha ${ }^{1}$, Huseyin Arslan ${ }^{1,2}$ \\ ${ }^{1}$ Department of Electrical and Electronics Engineering, Istanbul Medipol University, Istanbul, 34810, Turkey \\ ${ }^{2}$ Department of Electrical Engineering, University of South Florida, Tampa, FL, 33620, USA
}

NOTE: Corresponding author: Ahmet Yazar (ayazar@medipol.edu.tr)

\begin{abstract}
The upcoming sixth generation (6G) communications systems are expected to support an unprecedented variety of applications, pervading every aspect of human life. It is clearly not possible to fulfill the service requirements without actualizing a plethora of flexible options pertaining to the key enabler technologies themselves. At that point, this work presents an overview of the potential $6 G$ key enablers from the flexibility perspective, categorizes them, and provides a general framework to incorporate them in the future networks. Furthermore, the role of artificial intelligence and integrated sensing and communications as key enablers of the presented framework is also discussed.
\end{abstract}

Keywords - 6G, adaptive, artificial intelligence, cognitive radio, dynamic, flexibility, sensing.

\section{INTRODUCTION}

Following the successful standardization of the Fifth Generation (5G) networks worldwide, academia and industry have started to turn their attention to the next generation of wireless communications networks [1]. At present, there are more than 100 research papers regarding the Sixth Generation (6G) of wireless communications. There is no doubt that new visions and perspectives will continue to be developed in the coming years. However, despite all these efforts, current literature lacks gathering the distinctive features of $6 \mathrm{G}$ under a single broad umbrella.

The evolution of cellular communications through different generations from the Radio Access Technology (RAT) perspective is shown in Table 1 . The number of capabilities for newer cellular generations increases as a result of the need to meet diversified requirements. Flexibility ${ }^{1}$, where it is defined as the capability of making suitable choices out of available options depending on the internal and external changes, of the communications systems eventually evolves with an increasing number of new options. In this context, Fig. 2 provides a concise flexibility analysis for different generations of cellular communications considering the features in Table 1.

The Second Generation (2G) systems have paved the way for flexibility in communications systems by means of multiple frequency reuse options, adaptive equalization, and dynamic channel allocation. The Third Generation (3G) and the Fourth Generation (4G) cellular systems have incorporated voice communications with data communications. Additionally, Code Division Multiplexing (CDMA) and Orthogonal Frequency Division Multiplexing

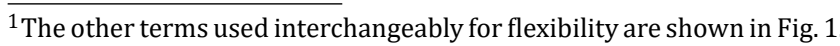

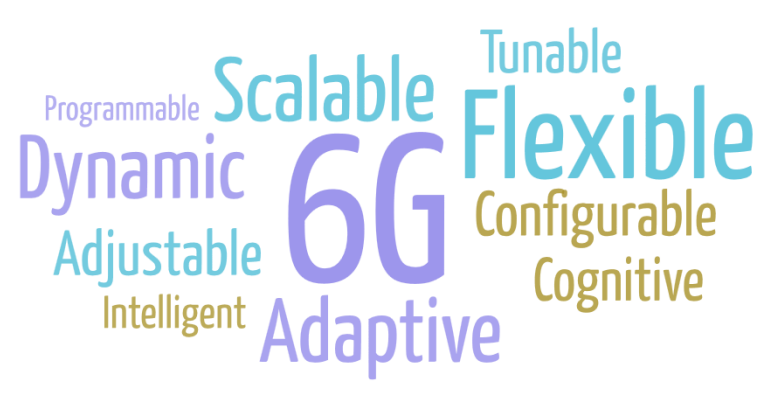

Fig. 1 - Flexibility terms.

(OFDM) have provided flexibility in terms of multiplexing, rate adaptation and interference management via the exploitation of different spreading factors and the multidimensional resource utilization, respectively.

The introduction of various services with rich requirement sets under $5 \mathrm{G}$ has revealed the need for a flexible network that can simultaneously meet diverse requirements. $5 \mathrm{G}$ has given a start for flexible wireless communications by the accommodation of different technologies. To exemplify, the coexistence of multinumerology in a single frame has been adopted during standardization meetings. In a given network, achieving flexibility is mainly dependent on three capabilities 1) awareness, 2) availability of a rich set of technology options, and 3) adaptation \& optimization. On this basis, although the flexibility perspective has been broadened in 5G systems with respect to previous generations, the existing technology options are not enough to reach all the goals of $5 \mathrm{G}$ networks. Additionally, it is expected that 6G networks will put further pressure on service providers due to emerging applications and use cases corresponding to new sets of requirements. Therefore, 6G systems need to extend the current flexibility by (1) exploring the awareness for the different aspects of the whole communications network and environment 


\begin{tabular}{|c|c|c|c|c|c|c|c|c|c|c|c|c|}
\hline Features/ Generations & \multicolumn{3}{|c|}{$1 \mathrm{G}$} & \multicolumn{3}{|c|}{$2 \mathrm{G}$} & \multicolumn{2}{|c|}{$3 G$} & \multicolumn{2}{|c|}{ 4G } & \multicolumn{2}{|c|}{$5 \mathrm{G}$} \\
\hline \multirow{5}{*}{$\begin{array}{l}\text { Modulation } \\
\text { Options }\end{array}$} & \multirow{5}{*}{\multicolumn{3}{|c|}{$\mathrm{FM}$}} & \multirow{2}{*}{ GSM } & \multirow{2}{*}{\multicolumn{2}{|c|}{ GMSK }} & EDGE & 8PSK & \multirow{5}{*}{\multicolumn{2}{|c|}{$\begin{array}{c}\text { BPSK } \\
\text { QPSK } \\
16 \text { QAM } \\
64 \text { QAM }\end{array}$}} & \multirow{5}{*}{ NR } & \multirow{5}{*}{$\begin{array}{c}\text { BPSK, QPSK, } \\
\text { 16QAM, 64AAM, } \\
256 \text { QAM }\end{array}$} \\
\hline & & & & & & & CDMA 2000 & QPSK, OQPSK & & & & \\
\hline & & & & \multirow{3}{*}{ GPRS } & \multirow{3}{*}{\multicolumn{2}{|c|}{ GMSK }} & W-CDMA & QPSK, OQPSK & & & & \\
\hline & & & & & & & UMTS & QPSK & & & & \\
\hline & & & & & & & HSDPA & QPSK, 16 QAM & & & & \\
\hline \multirow{3}{*}{$\begin{array}{l}\text { Coding } \\
\text { Options }\end{array}$} & & & & \multicolumn{3}{|c|}{ Convolutional Coding } & \multicolumn{2}{|c|}{ Turbo Coding } & \multicolumn{2}{|c|}{ Turbo Coding } & \multicolumn{2}{|c|}{ LDPC } \\
\hline & & & & \multirow{2}{*}{\multicolumn{3}{|c|}{ Block Coding }} & \multirow{2}{*}{\multicolumn{2}{|c|}{ Convolutional Coding }} & \multirow{2}{*}{\multicolumn{2}{|c|}{ Convolutional Coding }} & \multirow{2}{*}{\multicolumn{2}{|c|}{$\begin{array}{l}\text { Block Coding } \\
\text { Polar Coding }\end{array}$}} \\
\hline & & & & & & & & & & & & \\
\hline $\begin{array}{l}\text { Modulation and Coding } \\
\text { Scheme (MCS) Options }\end{array}$ & & & & \multicolumn{3}{|c|}{ Fixed } & \multicolumn{2}{|c|}{ Limited MCS Options } & \multicolumn{2}{|c|}{ Medium Mcs Options } & & cs Options \\
\hline & & & & & red Latt & & & attice & & Lattice & & ve Lattice \\
\hline Waveform & & & & Fixed & Shape I & GMSK) & Fixed S & ape (RC) & Windowin & and Filtering & Windor & $\begin{array}{l}\text { aptive } \\
\text { gand Filtering }\end{array}$ \\
\hline Options & & & & Fixed Type & The sar & e for uplink & Fixed Type (Th & same for uplink & Uplink & SC-FDE & Uplink & OFDM, SC-FDE \\
\hline & & & & an & down & ink) & and $\mathrm{d}$ & vnlink) & Downlink & OFDM & Downlink & OFDM \\
\hline Multiple Accessing & & FDMA & & & & & & & Uplink & SC-FDMA & Uplink & SC-FDMA \\
\hline Options & & 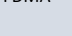 & & & 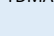 & & & & Downlink & OFDMA & Downlink & OFDMA \\
\hline Carrier Frequency & microwave & AMPS & $800 \mathrm{MHz}$ & microwave & GSM & $900 \mathrm{MHz}$ & microWave & $800 \mathrm{MHz}$ & microwave & $600 \mathrm{MHz}$ & microWave & $600 \mathrm{MHz}-6 \mathrm{GHz}$ \\
\hline Options & & NMT & $450 \mathrm{MHz}$ & & & $1800 \mathrm{MHz}$ & & $2.1 \mathrm{GHz}$ & & $2.5 \mathrm{GHz}$ & mmWave & $6 \mathrm{GHz}-300 \mathrm{GHz}$ \\
\hline Architecture Options & & siso & & & SISO & & & & & мо & & иімо \\
\hline Cell Planning & Freque & ency Reuse & & Frequency & Reuse & $-3,4,7,12$ & Frequen & Reuse - 1 & $\begin{array}{r}\text { Frequen } \\
\text { Fractional F } \\
\text { Soft Freg }\end{array}$ & $\begin{array}{l}\text { Reuse }-1 \\
\text { quency Reuse } \\
\text { necy Reuse }\end{array}$ & $\begin{array}{r}\text { Frequ } \\
\text { Fractionn } \\
\text { Soft } \mathrm{F} \\
\end{array}$ & $\begin{array}{l}\text { Ey Reuse - } 1 \\
\text { equency Reuse } \\
\text { uency Reuse }\end{array}$ \\
\hline & & & & & & & & & & ic & & OMP \\
\hline Options & & & & Mobile- & issisted & Hand-off & Soft & nd-off & Attem & to COMP & & RAN \\
\hline & & & & & & & & & 然 & 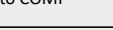 & & all Cell \\
\hline & & & & & & & & & & Multi-User & Freq. & Multi-User Diversity \\
\hline & & & & Freq. & Frequ & ency Hopping & Freq. & FHSS & . & Diversity & Space & MIMO \\
\hline Diversity & & & & & & & & & Space & MIMO & Space & Precoding \\
\hline Options & & & & & & & & & Space & Precoding & Space & Beamforming \\
\hline & & & & Time & & h Diversity & Time & DSSS & Space & Beamforming & Space & СомP \\
\hline Receiver Types & & & & & Iti-tap & & Rake & eceiver & A sing & Tap FDE & & e Tap FDE \\
\hline & & & & GSM & & KHz (8 slots) & CDMA & $1.25 \mathrm{MHz}$ & $1.25 \mathrm{Mr}$ & to $20 \mathrm{MHz}$ & & SWP \\
\hline Bandwidth & & & & & & & & & & & Carri & Iggregation \\
\hline Options & AMPS & & $\mathrm{kHz}$ & DAMPS & & $\mathrm{Hz}$ (3 slots) & WCDMA & $5 \mathrm{MHz}$ & $100 \mathrm{MHz}$ & & & umerology \\
\hline & & & & PDC & & $\mathrm{Hz}$ (3 slots) & TD-SCDMA & $1.6 \mathrm{MHz}$ & Agg & gation & License $A:$ & ed Access (LAA) \\
\hline
\end{tabular}

Table 1 - Increasing number of features for cellular generations.

using different sensing mechanisms including Artificial Intelligence (AI), (2) enriching technology options, and (3) providing optimum utilization of available options considering the awareness with practical sensing capabilities.

The work scopes of $6 \mathrm{G}$ publications in the literature are summarized in Table 2. A majority of the current $6 \mathrm{G}$ related studies attempt to identify the future applications and their key requirements [1,3-39]. Moreover, potential service types and application groups for $6 \mathrm{G}$ are analyzed in $[1,4-14,24-28]$ together with the prospective key requirements of $6 \mathrm{G}$ networks. Several works are focused on the key enabler technologies and concepts under $6 \mathrm{G}$ studies in detail $[1,3]$ or in general [4-13, 15-21]. Furthermore, specific technologies and concepts are also being pushed for $6 \mathrm{G}$ as described in [24-83]. These studies are revisited in the next two sections, however, it is seen that the flexibility perspective of the key enablers is not considered as a distinguishing feature for $6 \mathrm{G}$ systems in the literature.

In light of the aforementioned discussions, 6G networks require the redesign of cellular communications to provide extreme flexibility in all of its building blocks. Correspondingly, this paper elaborates the example flexibility aspects of potential 6G key enablers and provides a unique categorization of the related technologies and concepts. Moreover, a novel framework is proposed to gather the said enablers under an umbrella of a single ultra-flexible framework for 6G.

The rest of the paper is organized as follows: Section 2 gives a brief overview for the initial forecasts on $6 \mathrm{G}$ to explain the background for the necessity of a flexible perspective without examining all potential applications, re- 


\begin{tabular}{|c|c|c|c|c|c|c|}
\hline & \multicolumn{6}{|l|}{ †Flexibility } \\
\hline & $\begin{array}{l}\text { Infancy Stage of } \\
\text { Cellular } \\
\text { Communications }\end{array}$ & $\begin{array}{c}\text { Evolved Stage of } \\
\text { Cellular } \\
\text { Communications }\end{array}$ & $\begin{array}{l}\text { Infancy Stage of } \\
\text { High Speed } \\
\text { Data Rates }\end{array}$ & $\begin{array}{l}\text { Evolved Stage of } \\
\text { High Speed } \\
\text { Data Rates }\end{array}$ & $\begin{array}{l}\text { Infancy Stage of } \\
\text { Flexible } \\
\text { Communications } \\
\text { Platform }\end{array}$ & $\begin{array}{c}\text { Evolved Stage of } \\
\text { Flexible } \\
\text { Communications } \\
\text { Platform }\end{array}$ \\
\hline Transmitter & $\begin{array}{l}\text { Standard Defined - } \\
\text { No Flexibility }\end{array}$ & $\begin{array}{l}\text { Standard Defined - } \\
\text { Low Flexibility }\end{array}$ & $\begin{array}{l}\text { Standard Defined - } \\
\text { Medium Flexibility }\end{array}$ & $\begin{array}{l}\text { Standard Defined } \\
\text { High Flexibility }\end{array}$ & $\begin{array}{l}\text { Only Essential Rules } \\
\text { Standard Defined - } \\
\text { Very High Flexibility }\end{array}$ & $\begin{array}{l}\text { Only Essential Rules } \\
\text { Standard Defined - } \\
\text { Ultra Flexibility }\end{array}$ \\
\hline Channel & Random & Random & Random & Random & Random & Controlled \\
\hline Interference & Avoided & Avoided & Controlled & Controlled & $\begin{array}{l}\text { Intentionally Created } \\
\text { and Controlled }\end{array}$ & $\begin{array}{l}\text { Intentionally Created } \\
\text { and Controlled }\end{array}$ \\
\hline Receiver & No Flexibility & Low Flexibility & Medium Flexibility & High Flexibility & Very High Flexibility & Ultra Flexibility \\
\hline & & & & & & Generations \\
\hline & $1 G$ & $2 \mathrm{G}$ & $3 G$ & $4 G$ & $5 G$ & $6 \mathrm{G}$ \\
\hline
\end{tabular}

Fig. 2 - Flexibility analysis of the previous generations and 6G communications.

quirements, and service types. Flexibility discussions on the potential 6G key enablers are provided under a unique categorization rather than giving different details of these enablers in Section 3. The scope of Section 3 is limited to example flexibility aspects for the potential 6G key enablers. Next, a framework is proposed to increase interoperability of the $6 \mathrm{G}$ enablers in Section 4 . Finally, conclusions are drawn with several open issues in Section 5.

\section{A BRIEF OVERVIEW: FORECASTS ON 6G}

Identification of the future applications, requirements and possible service types is one of the primary objectives of the initial $6 \mathrm{G}$ research studies. Fig. 3 illustrates the basic relationship between these components. Mapping the potential future applications to the several requirements with different priorities is accepted as a first step in general. Next, these requirements are grouped under the service types in a reasonable manner. At the final stage, service types have unique requirement sets for the related application groups. In 5G systems, applications are considered under three service types including enhanced Mobile BroadBand (eMBB), Ultra-Reliable and Low-Latency Communications (URLLC), and massive Machine-Type Communications (mMTC) [84]. Among these, eMBB applications prioritize high throughput, capacity and spectral efficiency; mMTC prioritizes energy efficiency and massive connectivity while URLLC requires high reliability and low latency. For 6G systems, some of the initial studies inherently analyze the relations between the future applications and prioritized requirements to propose candidate service types [1,3-38].

The following list exemplifies potential 6G applications: drone and Unmanned Aerial Vehicle (UAV) networks, drone taxi, fully automated Vehicle-to-Everything (V2X), remote surgery, health monitoring, e-health, fully sen- sory Virtual Reality (VR) and Augmented Reality (AR), holographic conferencing, virtual education, virtual tourism, smart city, smart home, smart clothes, disaster and emergency management, and work-from-anywhere. This list can be longer with more applications in the upcoming years. Most of the aforementioned applications were originally envisioned for $5 \mathrm{G}$, however, they could not be practically realized. Therefore, it makes sense to address them first while developing the $6 \mathrm{G}$ networks.

General wireless communications requirements for the given application examples can be defined as: high data rate, high throughput, high capacity, high reliability, low latency, high mobility, high security, low complexity, high connectivity, long battery life, low cost, wide coverage, and more. The importance and priority of the requirements may change under different cases. Moreover, higher levels of performances need to be obtained in next generation systems while meeting the related requirements.

Since the requirement diversity is continuously increasing, more sophisticated service types are expected for 6G. Candidate service types are constituted by grouping applications with similar requirements. Examples ${ }^{2}$ can be given as Big Communications (BigCom), secure uRLLC (SuRLLC), Three-Dimensional Integrated Communications (3D-InteCom), Unconventional Data Communications (UCDC) in [11]; ultra-High-Speed-withLow-Latency Communications (uHSLLC) in [4]; LongDistance and High-Mobility Communications (LDHMC), Extremely Low-Power Communications (ELPC) in [5]; reliable eMBB; Mobile Broadband Reliable Low Latency Communication (MBRLLC), massive URLLC (mURLLC),

\footnotetext{
${ }^{2}$ Comprehensive discussions on these potential service types can be found in the given references.
} 


\begin{tabular}{c|c|c|c|c|c|c}
\hline Ref. & $\begin{array}{c}\text { Potential } \\
\text { Applications } \\
\text { and Key Re- } \\
\text { quirements }\end{array}$ & $\begin{array}{c}\text { Potential } \\
\text { Service Types } \\
\text { and } \\
\text { Application } \\
\text { Groups }\end{array}$ & $\begin{array}{c}\text { Focusing on } \\
\text { the Key } \\
\text { Enablers in } \\
\text { General }\end{array}$ & $\begin{array}{c}\text { Focusing on } \\
\text { the Key } \\
\text { Enablers in } \\
\text { Detail }\end{array}$ & $\begin{array}{c}\text { An Inclusive } \\
\text { Perspective } \\
\text { for the Key } \\
\text { Enablers }\end{array}$ & $\begin{array}{c}\text { The Future of } \\
\text { A Specific } \\
\text { Technology } \\
\text { or Concept }\end{array}$ \\
\hline This work & $\checkmark$ & $\checkmark$ & $\checkmark$ & & $\checkmark$ & \\
\hline$[1]$ & $\checkmark$ & $\checkmark$ & & $\checkmark$ & & \\
\hline$[3]$ & $\checkmark$ & & & $\checkmark$ & & \\
\hline$[4-13]$ & $\checkmark$ & $\checkmark$ & $\checkmark$ & & & \\
\hline$[14]$ & $\checkmark$ & $\checkmark$ & & & & \\
\hline$[15-21]$ & $\checkmark$ & & $\checkmark$ & & & \\
\hline$[22,23]$ & $\checkmark$ & & & & & \\
\hline$[24-28]$ & $\checkmark$ & $\checkmark$ & & & & \\
\hline$[29-39]$ & $\checkmark$ & & & & & $\checkmark$ \\
\hline$[40-83]$ & & & & & & $\checkmark$ \\
\hline
\end{tabular}

Table 2 - Scopes of 6 G publications in the literature.

Human-Centric Services (HCS), Multi-Purpose Services (MPS) in [7]. As it is seen from the names, some of the service types (e.g., SuRLLC, uHSLLC, reliable eMMB, MBRLLC, mURLLC, MPS) try to be more inclusive than the $5 \mathrm{G}$ service types to serve target applications. It is also possible to see more specific service types (e.g., BigCom, 3D-InteCom, UCDC, LDHMC, ELPC, HCS) in comparison with 5G.

The aforementioned applications/services envisioned for $6 \mathrm{G}$ illustrates the expected richness of its requirements. These diverse requirements necessitate an ultra-flexible perspective for the incorporation of key enabler technologies and concepts, described below, in future networks.

\section{ULTRA-FLEXIBLE PERSPECTIVE FOR 6G}

In this section, an inclusive categorization of promising key enablers is presented for 6G communications and their example flexibility aspects are discussed in detail. Then, several flexibility challenges are provided for 6G. Key enabler categories and their related subcategories are shown in Fig. 4. Many of these technologies are either superficially treated or not studied during $5 \mathrm{G}$ standardizations, such as Integrated Sensing and Communications (ISAC) and intelligent communications.

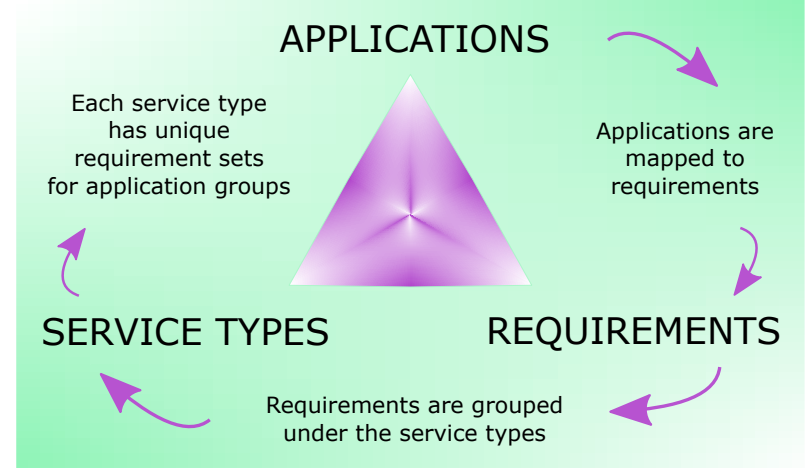

Fig. 3 - A basic relationship between the applications, requirements, and service types.
Although technologies placed in different categories can have overlapped regions, the given categorization differentiates these technologies regarding their flexibility aspects.

Table 3 provides a summary of the example flexibility options achieved by the different technologies. It is worthy to emphasize that the different key enablers have their own impact on the overall flexibility of the system. Ultimately all of them combine together to provide the complete infrastructure capable of realizing the flexible $6 \mathrm{G}$ vision that we aspire to achieve.

\subsection{Flexible Multi-Band Utilization}

The inclination of communications technologies towards high-frequency bands becomes more appealing due to the increased system capacity and throughput demands of cellular users. Furthermore, flexible usage of available frequency bands, depending on the user and service requirements, is envisioned to be an inherent characteristic of future wireless networks [74].

The millimeter Wave (mmWave) spectrum is starting to be exploited in 5G. It provides new benefits, such as multi-gigabit data rates and reduced interference, however, the use of mmWave bands in $5 \mathrm{G}$ is limited by the current International Mobile Telecommunications (IMT) regulations. In World Radiocommunication Conference 2019 (WRC-19), additional $17.25 \mathrm{GHz}$ of spectrum is identified for IMT, where only $1.9 \mathrm{GHz}$ of bandwidth was available before [85]. Therefore, it is expected that spectrum availability in these bands and consequently its flexible utilization will increase during the upcoming years [42]. Moreover, beyond $52.6 \mathrm{GHz}$ communications is one of the agenda items for 3GPP Release 17 [86].

Frequency bands from $100 \mathrm{GHz}$ to $3 \mathrm{THz}$ are envisioned as a candidate spectrum for $6 \mathrm{G}$ communications [40]. If $\mathrm{THz}$ communications is employed in $6 \mathrm{G}$, it promises a way 

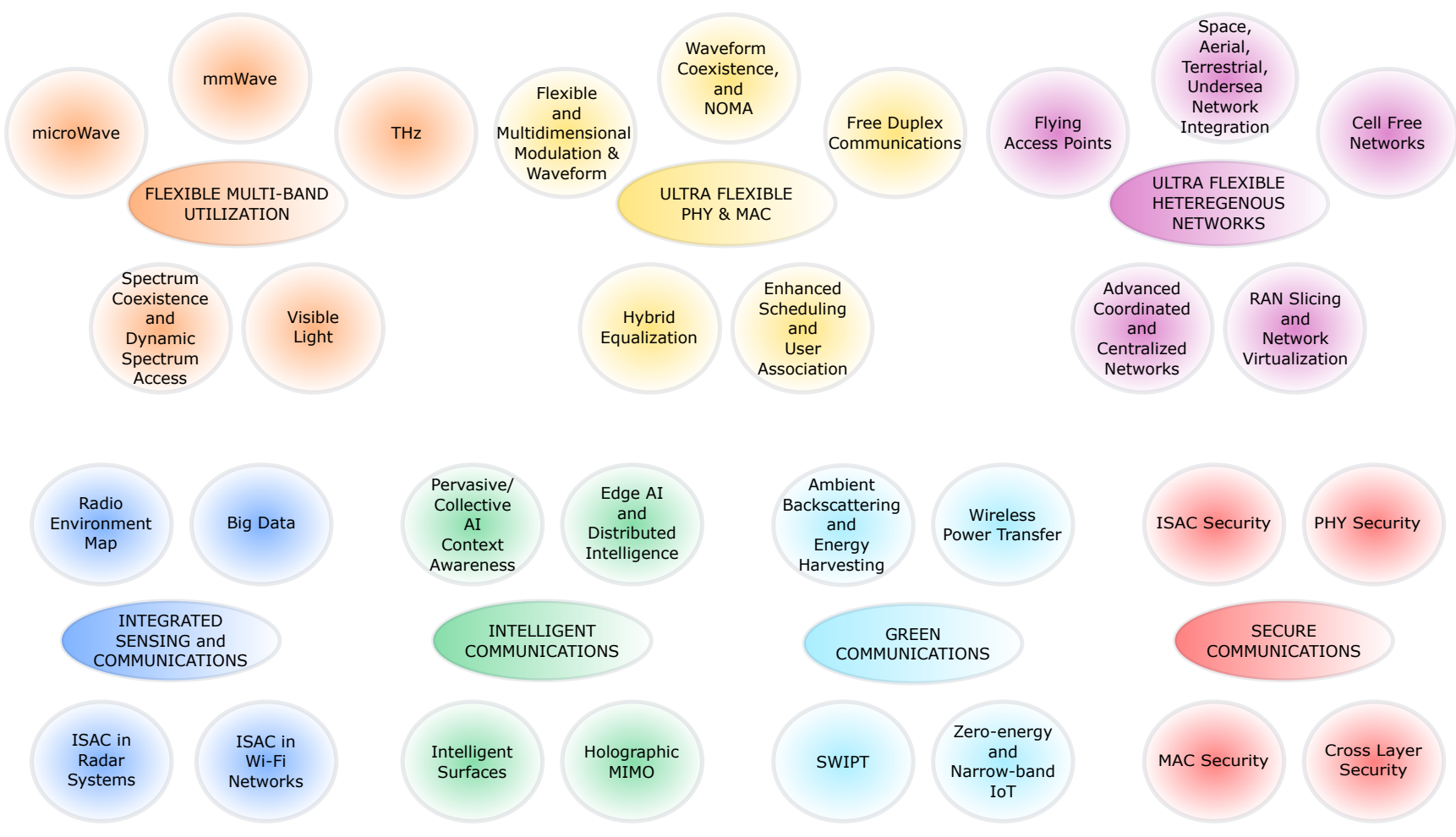

Fig. 4 - Categorization of the promising 6G key enablers under the ultra-flexible perspective.

of dealing with the spectrum scarcity issue by providing an additional degree of flexibility in assigning the most suitable frequency resources for given scenarios [46].

Apart from mmWave and $\mathrm{THz}$ communications, Visible Light Communications (VLC) also provides spectrum flexibility as a candidate key enabler for $6 \mathrm{G}$ networks [27, 52, 61]. Moreover, a new degree of freedom that is information source flexibility is exploited using visible light sources.

Spectrum coexistence is another important issue in need of flexible spectrum utilization $[50,74]$. Indeed, the coexistence of cellular communications, Wi-Fi, satellite networks, and radar systems is inevitable in the future due to both scarce resources and increasing growth in user demands. To exemplify, the coexistence of radar and cellular communications in mmWave frequency bands becomes more popular nowadays [87]. Moreover, the idea of Dynamic Spectrum Access (DSA) relies on the spectrum coexistence [56].

As it is seen, there are several aspects of flexible multiband utilization in 6G systems. Flexibility sources can be summarized under three main perspectives: 1) multiband flexibility, 2) information source flexibility, and 3) spectrum coexistence flexibility.

\subsection{Ultra-Flexible PHY and MAC}

One of the unique features of $5 G$, specifically in the context of PHY design, is the introduction of numerology concept where different configurations of the time-frequency lattice are used to address the varying requirements [88]. While the numerology concept paves the way for flexibility in beyond $5 \mathrm{G}$ networks, it is rather limited considering the competing nature of requirements expected for future 6G networks [25]. In addition to the standardized activities, the use of flexible Cyclic Prefix (CP) configurations (e.g., individual CP, common $\mathrm{CP}$, etc.) is explored to enhance the multi-numerology systems for $6 \mathrm{G}$ [89].

Taking one step beyond the use of different realizations of the same parent waveform as in 5G, multiple waveforms can be accommodated in a single frame for achieving $6 \mathrm{G}$ goals $[49,90]$. In line with this, multinumerology structures can be designed for promising alternative waveforms, that are more suitable for providing additional parameterization options. Having these options enhances flexibility in the PHY layer via increased adaptation capability for meeting a large number of requirements. Moreover, waveform coexistence in the same frame gives the opportunity to serve multiple networks such as radar sensing [91] and Wi-Fi communications together with 6G communications in a flexible manner. There are also several waveformdomain NOMA studies that exploit different resource utilization aspects in the literature [92-95]. Moreover, 
partial and full overlapping through available resources can also be employed while designing new generation NOMA techniques $[30,96]$. The waveform-domain NOMA concept provides an important flexibility by increasing the resource allocation possibilities in 6G networks [78]. Another flexibility aspect that can arise with $6 \mathrm{G}$ is the use of an alternate waveform domain rather than the conventional time-frequency lattice employed by $5 \mathrm{G}$ and older generations.

In addition to the waveform itself, there is a large number of new generation modulation options in the literature [97] and only a small set of them have appeared in the $5 \mathrm{G}$ standards. $6 \mathrm{G}$ can be enriched with the flexibility provided by these options, particularly Index Modulation (IM) based solutions [11]. This concept can even be extended to multiple domains to provide an additional degree of freedom [98]. Moreover, modulation techniques are adaptively designed considering the other key enablers such as Non-Orthogonal Multiple Access (NOMA) [99] and Reconfigurable Intelligent Surface (RIS) [45] for 6G.

Since the configuration of the PHY parameters is, to a large extent, controlled by the Medium Access Control (MAC) layer, it is imperative to develop the flexibility and adaptation capabilities of both layers simultaneously. Two important issues that require flexibility in PHY and MAC would be the "waveform parameter assignment" or "numerology scheduling" paradigm under the context of $5 \mathrm{G}$ multi-numerology systems $[25,100]$, where the MAC layer is responsible for assignment of parameters of the PHY signal. Similarly, adaptive guard utilization methods have been developed for the MAC layer [101-103] to control the new type of interferences in $5 \mathrm{G}$ systems. On this basis, it is expected that highly intelligent UE capabilities, and configurable network parameters, and flexible and efficient MAC designs will play a key role in $6 \mathrm{G}$ networks due to the expected increased diversity in service types and consequently requirements.

Example flexibility perspectives for ultra-flexible PHY and MAC technologies of potential 6G key enablers are given in Table 3.

\subsection{Ultra-Flexible Heterogeneous Networks}

Flying Access Points (FAPs) provide enhanced flexibility for network deployment by allowing dynamic (3-D) positioning of the nodes or even optimized trajectory planning for different objective functions $[47,55,59]$. The push in this direction occurred around the turn of the century [104], and was further empowered by projects, such as: 1) Google Loon project, 2) Facebook Aquila project, 3) ABSOLUTE project, 4) Matternet project, and 5) Thales Stratobus project. The integration of FAPs with the terrestrial network can be leveraged to provide coverage in disaster/emergency scenarios, connectivity for rural/isolated areas and capacity enhancement for temporarily crowded places (such as stadiums/concert venues) [77]. FAP-based networks are expected to be an important part of 6G not only for achieving deployment flexibility but also for having better wireless propagation provided by a high probability of Line of Sight (LOS) communications $[41,63]$.

In addition to the aerial and terrestrial networks, the integration of space (satellite) networks is another aspect of the flexible heterogeneous networks [54]. Space networks are also a promising solution for rural area communications [31]. They are employed for wireless backhaul communications in the previous cellular networks. However, space networks can also serve aerial user equipment such as drones and UAVs to increase coverage flexibility in 6G systems [72]. Moreover, undersea network integration with the other networks will be useful while serving naval platforms.

Although, the integration of different networks is ensured, the cell structures of these networks are changing. Cell-less or cell-free networks are one of the potential 6G concepts considering the network architecture richness $[105,106]$. User equipment connects to the network via multiple small cells in the cell-less networks. Cell-centric design is transformed into the user-centric system. Hence, it provides both handover-free communications and zero inter-cell interference. Cell-less networks may exploit a new dimension of network Multi-Input Multi-Output (MIMO) flexibility in 6G. As another network MIMO example, advanced coordinated and centralized networks [107] are addressed together with NOMA schemes for 6G communications [108,109]. These networks are called multi-cell NOMA. Flexibility comes with the number of the cells and architecture richness while exploiting other dimensions with NOMA.

From the network virtualization perspective, network slices are used in 5G to customize and optimize the network for service types or any other requirement sets [110-112]. Hence, the overall performance is increased by meeting different requirement sets with virtually privatized networks. Network slicing brings an important flexibility in $5 \mathrm{G}$ since it enables different network options under the same umbrella. The number of network slices can increase for $6 \mathrm{G}$ and there may be network slices for each user equipment. This user-centric network slicing architecture can provide full flexibility in the network layer.

The number of examples for the flexibility aspects of promising 6G heterogeneous networks can be increased with particular technologies and concepts such as blockchain systems [26,33] and quantum communications [29] in the future. 


\begin{tabular}{|c|c|c|}
\hline Key Enabler Categorization & Flexibility Aspect & Example Details \\
\hline \multirow{3}{*}{$\begin{array}{l}\text { Flexible Multi-Band } \\
\text { Utilization }\end{array}$} & Multi-band flexibility & microWave, mmWave, $\mathrm{THz}$, visible light \\
\hline & Information source flexibility & Radio signals, visible light \\
\hline & Spectrum coexistence flexibility & DSA, CR, and coexistence of cellular networks, Wi-Fi networks and radar systems \\
\hline \multirow{17}{*}{$\begin{array}{l}\text { Ultra-Flexible } \\
\text { PHY and MAC }\end{array}$} & Modulation-option flexibility & BPSK, QPSK, 16QAM, 64QAM, 256QAM, 1024QAM, etc. \\
\hline & Multi-domain modulation flexibility & IM (shape, interval, position, etc.), space, time, frequency, etc. \\
\hline & Multi-type coding & New types of LDPC, block coding, polar coding, etc. \\
\hline & MCS option flexibility & Ultra adaptive MCS \\
\hline & Multi-option waveform flexibility & Multiple numerologies for any specific waveform \\
\hline & Waveform processing flexibility & Adaptive windowing/filtering and the related configurable parameters \\
\hline & CP utilization flexibility & Individual and common CP utilizations \\
\hline & Adaptive guard utilization flexibility & Flexible guards for multi-waveform and multi-numerology designs \\
\hline & Multi-waveform flexibility & Waveform coexistence in the same frame \\
\hline & Multi-network multi-waveform flexibility & Waveform coexistence for cellular and Wi-Fi networks with radar sensing \\
\hline & Multi-domain NOMA flexibility & Partial and fully overlapped resources with waveform-domain NOMA \\
\hline & Multi-domain waveform flexibility & Alternative lattice flexibility together with the time-frequency lattice \\
\hline & Multiple access flexibility & Fully flexible, both orthogonal and non-orthogonal \\
\hline & Receiver-type flexibility & Fully flexible, hybrid equalization \\
\hline & Bandwidth option flexibility & BWP, carrier aggregation, LAA, DSA, etc. \\
\hline & User association flexibility & Multiple options under heteregonous networks, flexible user parameter assignment \\
\hline & Channel access flexibility & GB transmission, GF transmission, and their coexistence over a resource pool \\
\hline \multirow{8}{*}{$\begin{array}{c}\text { Ultra-Flexible } \\
\text { Heterogenous Networks }\end{array}$} & Positioning flexibility of the access points & Flying access points can be positioned flexibly in the sky \\
\hline & Connection link and relaying flexibility & User equipment can connect to different type of access points \\
\hline & Altitude-based multi-network flexibility & Coexistence of space, HAP, terrestrial and undersea networks \\
\hline & Coverage flexibility & Rural area coverage with space and HAP networks \\
\hline & Network architecture flexibility & Ultra massive MIMO, small cell, D2D, relaying via different networks, etc. \\
\hline & Cell-free network flexibility & User-centric network designs, handover-free communications \\
\hline & Multi-cell flexibility & Network MIMO solutions, multi-cell NOMA, etc. \\
\hline & Network slice flexibility & Network slices for each user equipment, user-specific virtual networks \\
\hline \multirow{2}{*}{$\begin{array}{l}\text { Integrated Sensing and } \\
\text { Communications }\end{array}$} & Multi-system flexibility & All systems can collabarate with the wireless communications in different ways \\
\hline & Awareness flexibility & Awareness in spectrum, location, mobility, context, user, channel, interference, etc. \\
\hline \multirow{5}{*}{ Intelligent Communications } & Alternative solution flexibility & No need to get stuck on conventional algorithm designs \\
\hline & Edge computing flexibility & Signal and data processing at the edge nodes \\
\hline & Channel control flexibility & Different types of intelligent surfaces \\
\hline & Interference management flexibility & Interference management with the channel control \\
\hline & Softwarization flexibility & Programmable architecture options for holographic MIMO systems \\
\hline \multirow{2}{*}{ Green Communications } & Battery-free implementation flexibility & Removing battery limitations and constraints \\
\hline & Interference exploitation flexibility & Interference can be useful for the energy harvesting \\
\hline \multirow{2}{*}{ Secure Communications } & Multi-domain security flexibility & No need to get stuck on key sharing security mechanisms, complementary solutions \\
\hline & Wireless channel exploitation flexibility & PHY security methods exploit the characteristics of wireless channel \\
\hline
\end{tabular}

Table 3 - Example flexibility aspects for the key enabler categories.

\subsection{Integrated Sensing and Communications}

With the emphasis on use cases such as V2X communications in recent years, sensing has attained increased importance leading to the integration of these two applications [113]. However, the use of sensing is not limited to V2X or autonomous driving. Rather, if there is any observable data that can be utilized for the optimization or enhancement of the communications systems, it should be leveraged in 6G [114]. The information pertaining to the radio environment can be utilized in improving network deployment, optimizing user association, providing secure communications and so on. Hence, one of the unique novelties of $6 \mathrm{G}$ systems is the integration of many different sensor hardware with the heterogeneous communications networks as exemplified in Fig. 5.

While it might sound like a novel idea to some, Integrated 


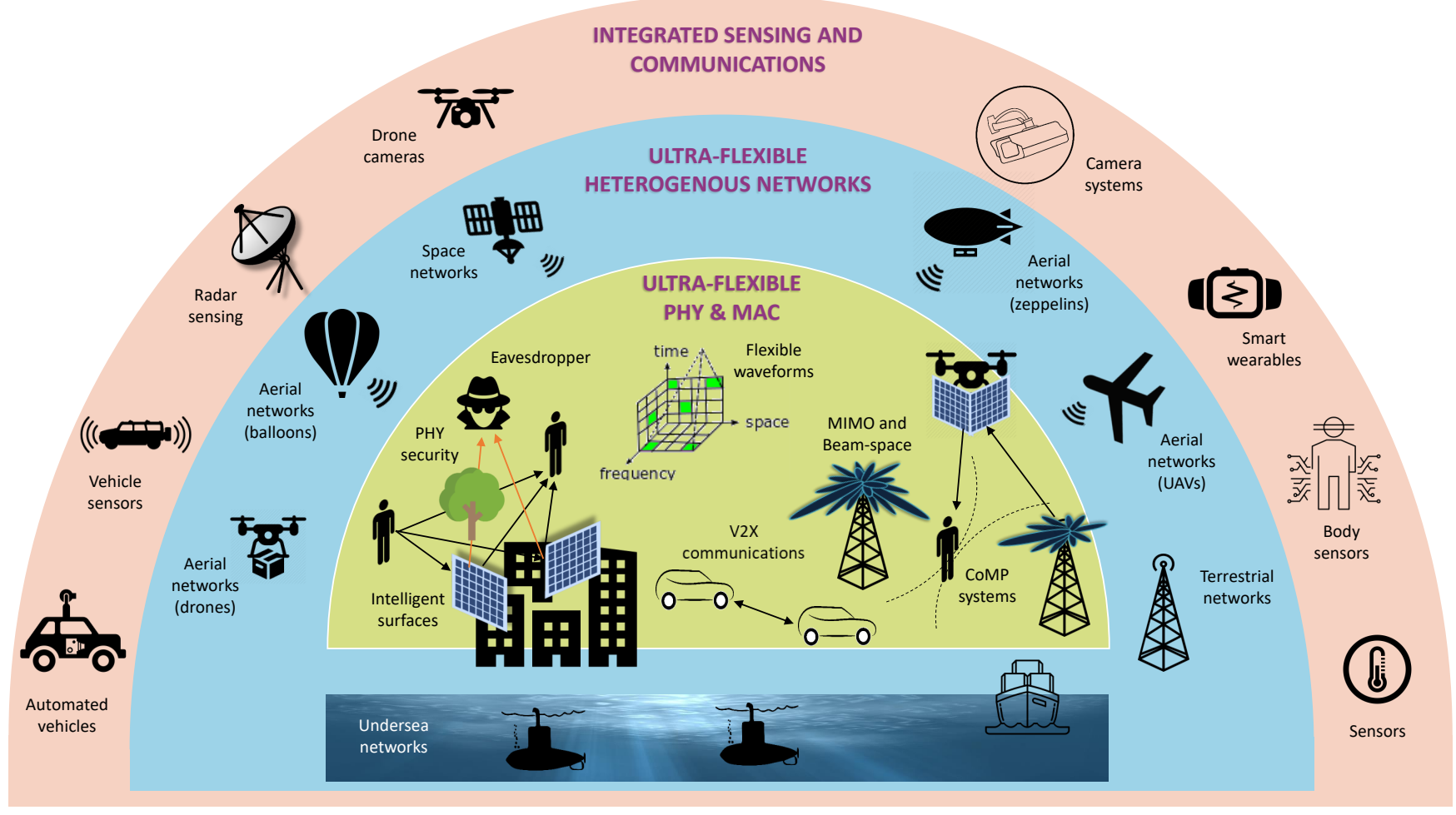

Fig. 5 - The integration of many different sensor hardware with the heterogeneous communications networks under 6G systems.

Sensing and Communications (ISAC) has been studied in different domains in the past. Cognitive Radio (CR) applications triggered the ISAC research on the last two decades. Spectrum sensing and awareness is one of the first application areas in the ISAC research [115]. Location awareness is exploited to improve the wireless communications system design in [116]. Satellite and drone images can be used to predict channel parameters [117]. Context-awareness is used to optimize network architectures in wireless communications [118]. ISAC systems are studied for radar sensing [91,119] and Wi-Fi network coexistence [120] in the literature. However, the complete list of sensing information that can be useful for the next generation cellular communications systems from the ISAC perspective has not yet been comprehensively studied [114].

A Radio Environment Map (REM) is a realization of the ISAC concept [121]. It is mainly used to obtain environmental information in the literature, however, for the next generation systems the REM concept will be generalized from environmental-awareness to completeawareness. REM may include all sensing information in a multi-dimensional manner for wireless communications networks. To exemplify, REM can be a specialized database for the ISAC. Therefore, the flexibility level of the ISAC systems can be determined by the dimensions in REMs. Each dimension in a REM increase the awareness, allowing better resource utilization. Moreover, control of the configurable options and parameters in different communications layers of $6 \mathrm{G}$ can be enhanced by more granular REM information.

The complete information and awareness of the environment comes at the cost of a high volume of data, variety of sources and significant processing $[80,82]$. This necessitates the use of big-data processing techniques [122]. A significant challenge, however, in this regard is the overhead of data exchange between the sensing and processing nodes. A centralized solution might not be suitable in such scenarios, rendering the use of edge-computing imperative, particularly for low-latency use cases. Moreover, the usage of Artificial Intelligence (AI) solutions can be helpful while processing big-data at the edge nodes.

\subsection{Intelligent Communications}

The usage of AI in the communications society has increased in recent years. Several survey and tutorial papers are published on the usage of Machine Learning (ML) for wireless communications [34, 123-129]. AIaided design and optimization has even been leveraged for the flexible implementation options provided in $5 \mathrm{G}$ [25]. In many of the studies, AI is put at the center of $6 \mathrm{G}$ visions $[6,8,24,28,32,44,67,75,76,83]$ to complement the classical methods. Indeed, the use of $\mathrm{AI}$ is inevitable to incorporate intelligence in the future networks [130-132]. AI-aided methods can propose fast and efficient solutions in case enough data is available.

$\mathrm{AI}$ and ML also find a range of applications in ISAC and REM paradigms to extract information regarding the en- 
vironment from sensed data. A flexible communications system needs to benefit from the advantages of popular ML approaches such as reinforcement learning, deep learning, and edge computing [37, 48, 69,73]. Especially distributed intelligence (edge $\mathrm{AI}$ ) with edge computing is a promising paradigm for $6 \mathrm{G}$ communications [36]. The management of multi-band utilization, MAC layer control, heterogeneous and cell-less networks, and the ISAC systems cannot be done in an all centralized manner. Edge computing will play an important role at that point with the help of distributed intelligence so $6 \mathrm{G}$ big data can be processed at the edge nodes without being collected at a centralized network.

Intelligent networks are not limited to AI-aided concepts. For example, RIS technology is one of the most popular research topics nowadays $[133,134]$. Intelligent surfaces bring a new flexibility on the control of channel parameters [57]. In the past, a wireless channel was just an observable medium. However, it can be controlled at some level with new generation wireless systems. Interference management flexibility is increased by controlling capabilities of the wireless channel. These flexibility aspects also affect the technology designs in different communications layers [66, 71]. To exemplify, having a control capability in multipath propagation, such as controlling delay spread, Doppler spread and the number of multipath alleviates the constraints related to waveform design. RIS technology can also be considered as passive holographic MIMO surfaces if it is located closer to the transmitter and receiver antennas [53]. Additionally, it is possible to employ holographic MIMO surfaces as active elements. The active holographic MIMO surfaces work similar to massive MIMO but their softwarization flexibility is higher than the conventional MIMO systems [53].

\subsection{Green Communications}

While candidate 6G key enablers are increasing the flexibility in different domains, new architectural changes of 6G should support energy efficiency and green communications $[43,64,70]$. Zero-energy Internet of Things (IoT) is one of the most important concepts since ultra low-power wireless communications is necessary for $6 \mathrm{G}$ connectivity [51]. In this context, Radio Frequency (RF) energy harvesting is studied with ambient backscatter technology for 6G communications $[135,136]$. Thus, low-power wireless systems can obtain their energy from the available high-power radio waves. Backscatter communications enables energy harvesting, simplifying the implementation of zero-energy IoT designs. Provision of rich options for energy-efficiency promises fulfilment of energy requirement variations belonging to different applications. Within this direction, the Symbiotic Radio (SR) concept offers highly reliable backscattering communications together with mutualism spectrum sharing $[137,138]$.
It is also possible to benefit from Wireless Power Transfer (WPT) while designing zero-energy IoT systems [139]. Under the WPT concept, Simultaneous Wireless Information and Power Transfer (SWIPT) is the most popular technology that may be a candidate for $6 \mathrm{G}$ networks [60, 140]. SWIPT designs are also used for interference exploitation purposes [141] since interference can be useful for energy harvesting. Transformation of interference into an energy source introduces another flexibility perspective.

\subsection{Secure Communications}

With applications such as eHealth, online banking, and autonomous driving etc., wireless communications promises to be an enabler of innumerable sensitive applications utilizing private data. However, the broadcast nature of wireless communications makes it vulnerable to several security threats such as eavesdropping, impersonation, and jamming. In order to ensure security of such applications, PHY Layer Security (PLS) is an emerging solution that has the capability to complement the conventional cryptography-based security techniques. In fact, PLS is more suited for the increased heterogeneity and power/processing restrictions of future wireless networks since it exploits the characteristics of the wireless channel and PHY properties associated with the link such as noise, fading, interference, and diversity [142]. It is also possible to increase this flexibility by designing cross-layer security algorithms with the PHY and MAC layer [143]. In several 6G papers, secure communications is discussed as one of the main topics [8, 35, 44, 81]. PHY and cross-layer security concepts are expected to play a critical role in 6G networks because of their capability to support joint design of security, reliability, and latency.

As discussed in the previous subsections, ISAC and REM concepts will be important enablers in 6G communications. However, a new security problem arises since there may be a large amount of confidential data for ISAC and REM concepts. In the literature, this problem is treated in [144] for ISAC security, and in [145] for REM security. Thus, there is a need for more secure communications options in 6G networks to meet new types of security requirements, especially for ISAC and REM concepts. Moreover, in order to tackle spoofing attacks, authentication at the physical layer by using features of channel and hardware impairments can also provide a fast, lightweight, and efficient alternative for crypto-security for authentication in future wireless networks. Furthermore, the physical layer solution will also provide efficient robustness against jamming attacks using terrestrial and flying relay and other new multi-antenna-based solution. 


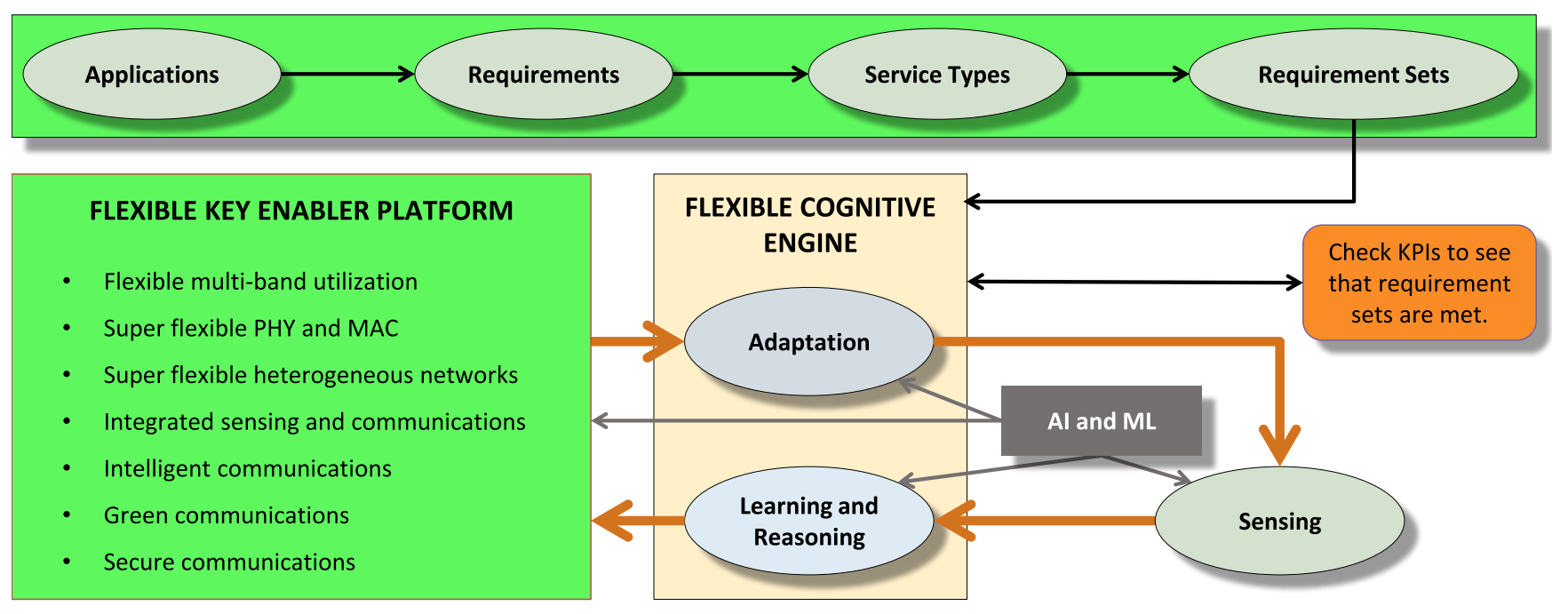

Fig. 6 - The proposed framework includes flexible key enabler platform and flexible cognitive engine. The flexible cognitive engine can be defined as a bridge between the requirements and potential technology options with the related configurations.

\subsection{Flexibility Challenges and Opportunities in $6 \mathrm{G}$}

The exemplified key enablers show that $6 \mathrm{G}$ will have many different flexibility options while $5 \mathrm{G}$ systems have limited flexibilities. However, each flexibility is coming with unique challenges. In other words, flexibility opportunities bring new challenges for the 6G networks.

For flexible multi-band utilization, operating the cellular system at multiple frequency bands needs advanced front-end hardware. Additionally, spectrum coexistence of different networks causes new interference problems. If the flexibility challenges on the PHY and MAC layer are investigated, one of the most important problems is the necessity of a flexible waveform system. At that point, either a single but an ultra-flexible waveform can be designed or multiple waveforms can be employed in the same frame. Designing a single waveform to meet all types of requirements did not work for $5 \mathrm{G}$ networks. It will be more difficult for $6 \mathrm{G}$ with more types of requirements. Moreover, waveform coexistence in the same frame causes new interferences (like inter-numerology interference in 5G). Similarly, partial and fully overlapped NOMA systems have the same interference problem. Control and mitigation of these interferences is expected as another challenge.

Flexibility challenges for heterogeneous networks can be exemplified with the developing optimal positioning and relaying algorithms for flying access points. In addition to these algorithms, interference management during the coexistence of different networks is necessary. As another challenge, network MIMO structures provide multi-cell flexibility, however, large amounts of data need to be transferred at the backhaul systems and the amount of burden increases.

As discussed in the previous subsections, ISAC systems can include multiple systems together with the communications networks. Generally, the amount of sensing information increases in parallel to awareness capabilities. However, processing the sensing information causes computational burdens. Additionally, investigating the ways of exploiting this information to enrich the communications systems is another important challenge.

For the flexibility challenges of intelligent communications, first of all, an efficient work distribution between conventional and ML methods is required. A large data sets and useful features need to be developed to make ML mechanisms more functional. Additionally, edge computing algorithm structures should be designed to reduce the workload at transmission points.

If we summarize the challenges and opportunities, the following items can be listed:

- Need for a rich set of algorithms and techniques at different layers of the protocol stack that are optimized for different applications with their own requirements.

- Integration of these rich sets of algorithms into the flexibility framework with minimal overhead and complexity.

- Development of techniques that allows flexibility with a simple parameter change without significantly impacting the rest of the system design.

- Integration of AI and ML techniques to solve complex system problems together with the classical model based approaches. AI/ML can be applied in different parts of our proposed framework, i.e. it can be applied for better sensing and learning, or for optimal use of the given set of algorithms and approaches, or developing better solutions in the transmission, reception, and modeling of the system. 
Therefore, there is a need for general frameworks and mechanisms to ease dealing with these challenges all together. Within this direction, an example framework is proposed in the next section.

\section{ULTRA-FLEXIBLE 6G FRAMEWORK}

Gathering together all potential 6G enablers in a flexible framework is an important challenge. Therefore, this section brings the above-mentioned flexible perspectives for the key enabler technologies and concepts under the umbrella of a single ultra-flexible framework for 6G. Here, it is important to realize that the presence of flexible options in itself is not enough to render a network intelligent. Rather, it needs the capability to make best use of the available options. Therefore, some sort of intelligence or cognition is imperative in future wireless networks. Keeping this in mind, the proposed framework has the following primary components: 1) Flexible key enabler platform (like an advanced Mitola radio), 2) flexible cognitive engine, and 3) flexibility performance indicators. Fig. 6 illustrates how these different components are interconnected within the framework. The key points of this framework can be summarized as follows:

1. New technologies should be integrated into communications standards via a flexible key enabler platform without waiting for ten years.

2. Key enabler technologies should work together in an optimal flexibility to meet different requirements. Therefore, a flexible cognitive engine can make an optimization between different flexibility aspects.

3. The amount of flexibility needs to be measured while making an optimization. Hence, developing new flexibility performance indicators is necessary.

The previous cellular communications generations were standardized approximately ten years apart. From a different point of view, it took about a decade for the available technologies to be included in the cellular standards. Waiting up to ten years to benefit from an available technology does not make sense if it is possible to develop a platform that hosts different technologies flexibly. For now, we need to tolerate the limited flexibility of $5 \mathrm{G}$ technologies for the next decade. However, an advanced Mitola radio can work like a smart phone that has installable and updateable software. We call this radio a flexible key enabler platform. In this concept, the platform has the ability to have new key enabler technologies by a softwarization. Thus, the flexibility level of the wireless communications system can be enhanced with new technologies and the related updates.

As it is shown in Fig. 6, each technology can bring different perspectives to the overall flexibility. There is a need for a multi-objective optimization unit to control all configurable and flexible aspects of the enablers in the flexible key enabler platform. This engine can be designed in an AI-aided manner to optimize the key enabler flexibilities jointly. An optimum work distribution should be done for the flexible configurations of key enablers to meet all the system requirements in the most efficient way. At the end, all system requirements should be met optimally. The flexible cognitive engine will guarantee this optimization by the help of Key Performance Indicators (KPIs) that show the success while meeting requirements. This flexibility optimizer considers also complexity requirements while operating the system.

ISAC technologies will be an important part of 6G technologies as discussed in the previous section. Any sensing information can be exploited to make the wireless communications more effective. The flexible cognitive engine can give decisions with more available information while meeting different requirements and handling with several impairments and constraints. Sensing information increases the awareness and controlling capabilities of the system. To provide these capabilities, AI tools in the flexible cognitive engine provide useful and unnoticeable relationships without heuristic designs and theoretical analysis. Hence, the flexible cognitive engine needs three important elements while optimizing the flexibility level with key enablers: 1) Sensing information to increase awareness and controlling capabilities, 2) AI tools to increase the functionality and effectiveness of sensing information, and 3) KPIs to monitor the overall system.

KPIs are needed to measure several performances of the communications system. One of these KPIs can be the flexibility performance indicator so that the achieved flexibility can be quantified. It is difficult to decide on a specific flexibility performance indicator because there are many different flexibility perspectives as shown in Table 3. This indicator can be technology-specific and require separate metrics for different technology categories. 6G networks will need flexibility indicators similar to the other KPIs such as spectral efficiency and reliability. Generally, the current key enabler technologies are not designed to be called flexible technologies. Flexibility aspects of these key enablers are described mostly based on the inferences. In ideal conditions, 6G technologies need to be designed considering the flexibility perspective as one of the key criteria. At that point, flexibility performance indicators should be employed to quantify the advantages and disadvantages of new designs in both the PHY and MAC layer.

\section{CONCLUSION}

$5 \mathrm{G}$ systems were characterized by diverse applications and requirements. $6 \mathrm{G}$ is expected to continue in the same vein by enriching the application fabric even further. Fulfilling such a wide variety of use cases is not possible unless flexibility is incorporated in the promising key enabling technologies for the future networks. Driven by 
this, we have presented example flexibility aspects for the potential 6G key enablers under a unique categorization.

We believe that 6G should be approached with flexibility at its primary design criterion. Flexibility aspects of the potential key enablers need to play a leading role in the design stages of $6 \mathrm{G}$ systems. To this end, we have presented a general framework comprising of the aforementioned flexible key enablers, empowered by a flexible cognitive engine and supported by different aspects of sensing and AI. We believe that the presence of flexible options is imperative but only that is not enough to support the future applications. The ability to extract information regarding the operating environment and making related intelligent decisions are the way forward in the wireless communications realm. The best possible utilization of the flexibility offered by the key enablers is determined with this vision.

The realization of a flexible key enabler platform like the one mentioned above is, however, not straightforward. It requires the methods capable of performing efficient multi-objective optimization to address the various competing applications requirements. Furthermore, quantifying the flexibility by proposing novel performance indicators also remains a significant challenge on the way to ensure a fully-functional flexible, cognitive wireless communications network.

\section{ACKNOWLEDGEMENT}

The authors would like to thank Muhammad Sohaib J. Solaija for his valuable comments and suggestions to improve the quality of the paper.

\section{REFERENCES}

[1] I. F. Akyildiz, A. Kak and S. Nie, "6G and Beyond: The Future of Wireless Communications Systems," in IEEE Access, vol. 8, pp. 133995-134030, 2020, doi: 10.1109/ACCESS.2020.3010896.

[2] H. Arslan and E. Basar, "Flexible and Cognitive Radio Access Technologies for 5G and Beyond," IET, 2020.

[3] L. Bariah et al., "A Prospective Look: Key Enabling Technologies, Applications and Open Research Topics in 6G Networks," in IEEE Access, vol. 8, pp. 174792174820, 2020, doi: 10.1109/ACCESS.2020.3019590.

[4] B. Zong, C. Fan, X. Wang, X. Duan, B. Wang and J. Wang, "6G Technologies: Key Drivers, Core Requirements, System Architectures, and Enabling Technologies," in IEEE Vehicular Technology Magazine, vol. 14, no. 3, pp. 18-27, Sept. 2019, doi: 10.1109/MVT.2019.2921398.

[5] Z. Zhang et al., "6G Wireless Networks: Vision, Requirements, Architecture, and Key Tech- nologies," in IEEE Vehicular Technology Magazine, vol. 14, no. 3, pp. 28-41, Sept. 2019, doi: 10.1109/MVT.2019.2921208.

[6] L. Zhang, Y. Liang and D. Niyato, "6G Visions: Mobile ultra-broadband, super internet-of-things, and artificial intelligence," in China Communications, vol. 16, no. 8, pp. 1-14, Aug. 2019, doi: 10.23919/JCC.2019.08.001.

[7] W. Saad, M. Bennis and M. Chen, "A Vision of 6G Wireless Systems: Applications, Trends, Technologies, and Open Research Problems," in IEEE Network, vol. 34, no. 3, pp. 134-142, May/June 2020, doi: 10.1109/MNET.001.1900287.

[8] G. Gui, M. Liu, F. Tang, N. Kato and F. Adachi, "6G: Opening New Horizons for Integration of Comfort, Security and Intelligence," in IEEE Wireless Communications, doi: 10.1109/MWC.001.1900516.

[9] M. Giordani, M. Polese, M. Mezzavilla, S. Rangan and M. Zorzi, "Toward 6G Networks: Use Cases and Technologies," in IEEE Communications Magazine, vol. 58, no. 3, pp. 55-61, Mar. 2020, doi: 10.1109/MCOM.001.1900411.

[10] J. F. Monserrat, D. Martin-Sacristan, F. Bouchmal, O. Carrasco, J. Flores de Valgas and N. Cardona, "Key Technologies for the Advent of the 6G," 2020 IEEE Wireless Communications and Networking Conference Workshops (WCNCW), Seoul, Korea (South), 2020, pp. 1-6, doi: 10.1109/WCNCW48565.2020.9124725.

[11] S. Dang, O. Amin, B. Shihada and M. Alouini, "What should 6G be?," in Nature Electronics, vol. 3, pp. 20$29,2020$.

[12] M. H. Alsharif et al., "Sixth Generation (6G) Wireless Networks: Vision, Research Activities, Challenges and Potential Solutions," in Symmetry, vol. 12, no. 4, pp. 1-21, 2020.

[13] F. Tariq, M. R. A. Khandaker, K. -K. Wong, M. A. Imran, M. Bennis and M. Debbah, "A Speculative Study on 6G," in IEEE Wireless Communications, vol. 27, no. 4, pp. 118-125, Aug. 2020, doi: 10.1109/MWC.001.1900488.

[14] G. Liu et al., "Vision, requirements and network architecture of 6G mobile network beyond 2030," in China Communications, vol. 17, no. 9, pp. 92-104, Sept. 2020, doi: 10.23919/JCC.2020.09.008.

[15] P. Yang, Y. Xiao, M. Xiao and S. Li, “6G Wireless Communications: Vision and Potential Techniques," in IEEE Network, vol. 33, no. 4, pp. 70-75, July/Aug. 2019, doi: 10.1109/MNET.2019.1800418. 
[16] S. Elmeadawy and R. M. Shubair, "6G Wireless Communications: Future Technologies and Research Challenges," 2019 International Conference on Electrical and Computing Technologies and Applications (ICECTA), Ras Al Khaimah, United Arab Emirates, 2019, pp. 1-5, doi: 10.1109/ICECTA48151.2019.8959607.

[17] G. Wikström et al., "Challenges and Technologies for 6G," 2020 2nd 6G Wireless Summit (6G SUMMIT), Levi, Finland, 2020, pp. 1-5, doi: 10.1109/6GSUMMIT49458.2020.9083880.

[18] Y. Yuan, Y. Zhao, B. Zong and S. Parolari, "Potential key technologies for 6G mobile communications," in Science China Information Sciences, vol. 63, no. 183301, pp. 1-19, 2020.

[19] L. U. Khan, I. Yaqoob, M. Imran, Z. Han and C. S. Hong, "6G Wireless Systems: A Vision, Architectural Elements, and Future Directions," in IEEE Access, vol. 8, pp. 147029-147044, 2020, doi: 10.1109/ACCESS.2020.3015289.

[20] A. Dogra, R. K. Jha and S. Jain, "A Survey on beyond $5 \mathrm{G}$ network with the advent of 6G: Architecture and Emerging Technologies," in IEEE Access, doi: 10.1109/ACCESS.2020.3031234.

[21] S. P. Rout, "6G Wireless Communication: Its Vision, Viability, Application, Requirement, Technologies, Encounters and Research," 2020 11th International Conference on Computing, Communication and Networking Technologies (ICCCNT), Kharagpur, India, 2020, pp. 1-8, doi: 10.1109/ICCCNT49239.2020.9225680.

[22] K. David and H. Berndt, "6G Vision and Requirements: Is There Any Need for Beyond 5G?," in IEEE Vehicular Technology Magazine, vol. 13, no. 3, pp. 7280, Sept. 2018, doi: 10.1109/MVT.2018.2848498.

[23] A. Mourad, R. Yang, P. H. Lehne and A. de la Oliva, "Towards 6G: Evolution of Key Performance Indicators and Technology Trends," 2020 2nd 6G Wireless Summit (6G SUMMIT), Levi, Finland, 2020, pp. 1-5, doi: 10.1109/6GSUMMIT49458.2020.9083759.

[24] M. J. Piran and D. Y. Suh, "Learning-Driven Wireless Communications, towards 6G," 2019 International Conference on Computing, Electronics \& Communications Engineering (iCCECE), London, United Kingdom, 2019, pp. 219-224, doi: 10.1109/iCCECE46942.2019.8941882.

[25] A. Yazar and H. Arslan, "A Waveform Parameter Assignment Framework for 6G With the Role of Machine Learning," in IEEE Open Journal of Vehicular Technology, vol. 1, pp. 156-172, 2020, doi: 10.1109/OJVT.2020.2992502.
[26] S. Aggarwal, N. Kumar and S. Tanwar, "Blockchain Envisioned UAV Communication Using 6G Networks: Open issues, Use Cases, and Future Directions," in IEEE Internet of Things Journal, doi: 10.1109/JIOT.2020.3020819.

[27] S. Ariyanti and M. Suryanegara, "Visible Light Communication (VLC) for 6G Technology: The Potency and Research Challenges," 2020 Fourth World Conference on Smart Trends in Systems, Security and Sustainability (WorldS4), London, United Kingdom, 2020, pp. 490-493, doi: 10.1109/WorldS450073.2020.9210383.

[28] K. B. Letaief, W. Chen, Y. Shi, J. Zhang and Y. A. Zhang, "The Roadmap to 6G: AI Empowered Wireless Networks," in IEEE Communications Magazine, vol. 57, no. 8, pp. 84-90, Aug. 2019, doi: 10.1109/MCOM.2019.1900271.

[29] S. J. Nawaz, S. K. Sharma, S. Wyne, M. N. Patwary and M. Asaduzzaman, "Quantum Machine Learning for 6G Communication Networks: State-of-the-Art and Vision for the Future," in IEEE Access, vol. 7, pp. 4631746350, 2019, doi: 10.1109/ACCESS.2019.2909490.

[30] Y. Al-Eryani and E. Hossain, "The D-OMA Method for Massive Multiple Access in 6G: Performance, Security, and Challenges," in IEEE Vehicular Technology Magazine, vol. 14, no. 3, pp. 92-99, Sept. 2019, doi: 10.1109/MVT.2019.2919279.

[31] E. Yaacoub and M. Alouini, "A Key 6G Challenge and Opportunity-Connecting the Base of the Pyramid: A Survey on Rural Connectivity," in Proceedings of the IEEE, vol. 108, no. 4, pp. 533-582, Apr. 2020, doi: 10.1109/JPROC.2020.2976703.

[32] Y. Chen, P. Zhu, G. He, X. Yan, H. Baligh and J. $\mathrm{Wu}$, "From Connected People, Connected Things, to Connected Intelligence," 2020 2nd 6G Wireless Summit (6G SUMMIT), Levi, Finland, 2020, pp. 1-7, doi: 10.1109/6GSUMMIT49458.2020.9083770.

[33] T. Hewa, G. Gür, A. Kalla, M. Ylianttila, A. Bracken and M. Liyanage, "The Role of Blockchain in 6G: Challenges, Opportunities and Research Directions," 2020 2nd 6G Wireless Summit (6G SUMMIT), Levi, Finland, 2020, pp. 1-5, doi: 10.1109/6GSUMMIT49458.2020.9083784.

[34] Y. Sun, J. Liu, J. Wang, Y. Cao and N. Kato, "When Machine Learning Meets Privacy in 6G: A Survey," in IEEE Communications Surveys \& Tutorials, doi: 10.1109/COMST.2020.3011561.

[35] H. Chen, K. Tu, J. Li, S. Tang, T. Li and Z. Qing, "6G Wireless Communications: Security Technologies and Research Challenges," 2020 International Conference on Urban Engineering and Management Science (ICUEMS), Zhuhai, China, 2020, pp. 592-595, doi: 10.1109/ICUEMS50872.2020.00130. 
[36] Y. Liu, X. Yuan, Z. Xiong, J. Kang, X. Wang and D. Niyato, "Federated learning for 6G communications: Challenges, methods, and future directions," in China Communications, vol. 17, no. 9, pp. 105-118, Sept. 2020, doi: 10.23919/JCC.2020.09.009.

[37] J. Du, C. Jiang, J. WANG, Y. Ren and M. Debbah, “Machine Learning for 6G Wireless Networks: CarryForward-Enhanced Bandwidth, Massive Access, and Ultrareliable/Low Latency," in IEEE Vehicular Technology Magazine, doi: 10.1109/MVT.2020.3019650.

[38] C. -X. Wang, J. Huang, H. Wang, X. Gao, X. You and Y. Hao, "6G Wireless Channel Measurements and Models: Trends and Challenges," in IEEE Vehicular Technology Magazine, doi: 10.1109/MVT.2020.3018436.

[39] Y. Zhou, L. Liu, L. Wang, N. Hui, X. Cui, J. Wu, Y. Peng, Y. Qi, C. Xing," "Service-aware 6G: An intelligent and open network based on the convergence of communication, computing and caching," in Digital Communications and Networks, vol. 6, no. 3, pp. 253-260, 2020.

[40] T. S. Rappaport et al., "Wireless Communications and Applications Above $100 \mathrm{GHz}$ : Opportunities and Challenges for 6G and Beyond," in IEEE Access, vol. 7, pp. 78729-78757, 2019, doi: 10.1109/ACCESS.2019.2921522.

[41] X. Huang, J. A. Zhang, R. P. Liu, Y. J. Guo and L. Hanzo, "Airplane-Aided Integrated Networking for 6G Wireless: Will It Work?," in IEEE Vehicular Technology Magazine, vol. 14, no. 3, pp. 84-91, Sept. 2019, doi: 10.1109/MVT.2019.2921244.

[42] L. Zhu, Z. Xiao, X. Xia and D. Oliver Wu, "MillimeterWave Communications With Non-Orthogonal Multiple Access for B5G/6G," in IEEE Access, vol. 7, pp. 116123-116132, 2019, doi: 10.1109/ACCESS.2019.2935169.

[43] T. Huang, W. Yang, J. Wu, J. Ma, X. Zhang and D. Zhang, "A Survey on Green 6G Network: Architecture and Technologies," in IEEE Access, vol. 7, pp. 175758175768, 2019, doi: 10.1109/ACCESS.2019.2957648.

[44] F. Tang, Y. Kawamoto, N. Kato and J. Liu, "Future Intelligent and Secure Vehicular Network Toward 6G: Machine-Learning Approaches," in Proceedings of the IEEE, vol. 108, no. 2, pp. 292-307, Feb. 2020, doi: 10.1109/JPROC.2019.2954595.

[45] E. Basar, "Reconfigurable Intelligent Surface-Based Index Modulation: A New Beyond MIMO Paradigm for 6G," in IEEE Transactions on Communications, vol. 68, no. 5, pp. 3187-3196, May 2020, doi: 10.1109/TCOMM.2020.2971486.

[46] M. Yu, A. Tang, X. Wang and C. Han, "Joint Scheduling and Power Allocation for 6G Terahertz Mesh Networks," 2020 International Conference on
Computing, Networking and Communications (ICNC), Big Island, HI, USA, 2020, pp. 631-635, doi: 10.1109/ICNC47757.2020.9049790.

[47] S. Zhang, H. Zhang and L. Song, "Beyond D2D: Full Dimension UAV-to-Everything Communications in 6G," in IEEE Transactions on Vehicular Technology, vol. 69, no. 6, pp. 6592-6602, June 2020, doi: 10.1109/TVT.2020.2984624.

[48] N. Kato, B. Mao, F. Tang, Y. Kawamoto and J. Liu, “Ten Challenges in Advancing Machine Learning Technologies toward 6G," in IEEE Wireless Communications, vol. 27, no. 3, pp. 96-103, June 2020, doi: 10.1109/MWC.001.1900476.

[49] X. Liu, T. Xu and I. Darwazeh, “Coexistence of Orthogonal and Non-orthogonal Multicarrier Signals in Beyond 5G Scenarios," 2020 2nd 6G Wireless Summit (6G SUMMIT), Levi, Finland, 2020, pp. 1-5, doi: 10.1109/6GSUMMIT49458.2020.9083780.

[50] S. Lagen, N. Patriciello and L. Giupponi, "Cellular and Wi-Fi in Unlicensed Spectrum: Competition leading to Convergence," 2020 2nd 6G Wireless Summit (6G SUMMIT), Levi, Finland, 2020, pp. 1-5, doi: 10.1109/6GSUMMIT49458.2020.9083786.

[51] N. H. Mahmood, H. Alves, O. A. López, M. Shehab, D. P. M. Osorio and M. Latva-Aho, "Six Key Features of Machine Type Communication in 6G," 2020 2nd 6G Wireless Summit (6G SUMMIT), Levi, Finland, 2020, pp. 15, doi: 10.1109/6GSUMMIT49458.2020.9083794.

[52] M. Katz and I. Ahmed, "Opportunities and Challenges for Visible Light Communications in 6G," 2020 2nd 6G Wireless Summit (6G SUMMIT), Levi, Finland, 2020, pp. 1-5, doi: 10.1109/6GSUMMIT49458.2020.9083805.

[53] C. Huang et al., "Holographic MIMO Surfaces for 6G Wireless Networks: Opportunities, Challenges, and Trends," in IEEE Wireless Communications, doi: 10.1109/MWC.001.1900534.

[54] C. Liu, W. Feng, Y. Chen, C. Wang and N. Ge, "Cell-Free Satellite-UAV Networks for 6G Wide-Area Internet of Things," in IEEE Journal on Selected Areas in Communications, doi: 10.1109/JSAC.2020.3018837.

[55] H. Hashida, Y. Kawamoto and N. Kato, "Intelligent Reflecting Surface Placement Optimization in Air-Ground Communication Networks Toward 6G," in IEEE Wireless Communications, doi: 10.1109/MWC.001.2000142.

[56] R. K. Saha, "Licensed Countrywide Full-Spectrum Allocation: A New Paradigm for Millimeter-Wave Mobile Systems in 5G/6G Era," in IEEE Access, vol. 8, pp. 166612-166629, 2020, doi: 10.1109/ACCESS.2020.3023342. 
[57] J. A. Hodge, K. V. Mishra and A. I. Zaghloul, "Intelligent Time-Varying Metasurface Transceiver for Index Modulation in 6G Wireless Networks," in IEEE Antennas and Wireless Propagation Letters, doi: 10.1109/LAWP.2020.3025333.

[58] A. Celik, A. Chaaban, B. Shihada and M. -S. Alouini, "Topology Optimization for 6G Networks: A Network Information-Theoretic Approach," in IEEE Vehicular Technology Magazine, doi: 10.1109/MVT.2020.3017152.

[59] M. Kishk, A. Bader and M. -S. Alouini, "Aerial Base Station Deployment in 6G Cellular Networks Using Tethered Drones: The Mobility and Endurance Tradeoff," in IEEE Vehicular Technology Magazine, doi: 10.1109/MVT.2020.3017885.

[60] W. Lu et al., "SWIPT Cooperative Spectrum Sharing for 6G-Enabled Cognitive IoT Network," in IEEE Internet of Things Journal, doi: 10.1109/JIOT.2020.3026730.

[61] N. Chi, Y. Zhou, Y. Wei and F. Hu, "Visible light communication in 6G: Advances, challenges, and prospects," in IEEE Vehicular Technology Magazine, doi: 10.1109/MVT.2020.3017153.

[62] J. Liu, W. Liu, X. Hou, Y. Kishiyama, L. Chen and T. Asai, "Non-Orthogonal Waveform (NOW) for 5G Evolution and 6G," 2020 IEEE 31st Annual International Symposium on Personal, Indoor and Mobile Radio Communications, London, United Kingdom, 2020, pp. 1-6, doi: 10.1109/PIMRC48278.2020.9217361.

[63] A. Vanelli-Coralli, A. Guidotti, T. Foggi, G. Colavolpe and G. Montorsi, "5G and Beyond 5G Non-Terrestrial Networks: trends and research challenges," 2020 IEEE 3rd 5G World Forum (5GWF), Bangalore, India, 2020, pp. 163-169, doi: 10.1109/5GWF49715.2020.9221119.

[64] L. Zhen, A. K. Bashir, K. Yu, Y. D. Al-Otaibi, C. H. Foh and P. Xiao, "Energy-Efficient Random Access for LEO Satellite-Assisted 6G Internet of Remote Things," in IEEE Internet of Things Journal, doi: 10.1109/JIOT.2020.3030856.

[65] S. Liao, J. Wu, J. Li and K. Konstantin, "InformationCentric Massive IoT based Ubiquitous Connected VR/AR in 6G: A Proposed Caching Consensus Approach," in IEEE Internet of Things Journal, doi: 10.1109/JIOT.2020.3030718.

[66] R. Alghamdi et al., "Intelligent Surfaces for 6G Wireless Networks: A Survey of Optimization and Performance Analysis Techniques," in IEEE Access, doi: 10.1109/ACCESS.2020.3031959.

[67] H. Yang, A. Alphones, Z. Xiong, D. Niyato, J. Zhao and $\mathrm{K}$. Wu, "Artificial Intelligence-Enabled
Intelligent 6G Networks," in IEEE Network, doi: 10.1109/MNET.011.2000195.

[68] H. Huang, S. Hu, T. Yang and C. W. Yuan, "Full Duplex Non-orthogonal Multiple Access with Layersbased Optimized Mobile Relays Subsets Algorithm in B5G/6G Ubiquitous Networks," in IEEE Internet of Things Journal, doi: 10.1109/JIOT.2020.3033553.

[69] Y. Liu, X. Wang, G. Boudreau, A. B. Sediq and H. Abouzeid, "A Multi-Dimensional Intelligent Multiple Access Technique for 5G Beyond and 6G Wireless Networks," in IEEE Transactions on Wireless Communications, doi: 10.1109/TWC.2020.3032631.

[70] A. Mukherjee, P. Goswami, M. A. Khan, L. Manman, L. Yang and P. Pillai, "Energy Efficient Resource Allocation strategy in Massive IoT for Industrial 6G Applications," in IEEE Internet of Things Journal, doi: 10.1109/JIOT.2020.3035608.

[71] I. Yildirim, A. Uyrus and E. Basar, "Modeling and Analysis of Reconfigurable Intelligent Surfaces for Indoor and Outdoor Applications in Future Wireless Networks," in IEEE Transactions on Communications, doi: 10.1109/TCOMM.2020.3035391.

[72] S. Wan, J. Hu, C. Chen, A. Jolfaei, S. Mumtaz and Q. Pei, "Fair-Hierarchical Scheduling for Diversified Services in Space, Air and Ground for 6G-Dense Internet of Things," in IEEE Transactions on Network Science and Engineering, doi: 10.1109/TNSE.2020.3035616.

[73] S. Han et al., "Artificial-Intelligence-Enabled Air Interface for 6G: Solutions, Challenges, and Standardization Impacts," in IEEE Communications Magazine, vol. 58, no. 10 , pp. $73-79$, Oct. 2020 , doi: 10.1109/MCOM.001.2000218.

[74] M. Matinmikko-Blue, S. Yrjölä and P. Ahokangas, "Spectrum Management in the 6G Era: The Role of Regulation and Spectrum Sharing," 2020 2nd 6G Wireless Summit (6G SUMMIT), Levi, Finland, 2020, pp. 1-5, doi: 10.1109/6GSUMMIT49458.2020.9083851.

[75] R. Shafin, L. Liu, V. Chandrasekhar, H. Chen, J. Reed and J. C. Zhang, "Artificial IntelligenceEnabled Cellular Networks: A Critical Path to Beyond-5G and 6G," in IEEE Wireless Communications, vol. 27, no. 2, pp. 212-217, Apr. 2020, doi: 10.1109/MWC.001.1900323.

[76] J. Zhu, M. Zhao, S. Zhang and W. Zhou, "Exploring the road to $6 \mathrm{G}$ : $\mathrm{ABC}$ - foundation for intelligent mobile networks," in China Communications, vol. 17, no. 6, pp. 51-67, June 2020, doi: 10.23919/JCC.2020.06.005.

[77] V. Ziegler, H. Viswanathan, H. Flinck, M. Hoffmann, V. Räisänen and K. Hätönen, "6G Architecture to Connect the Worlds," in IEEE Access, vol. 8, pp. 173508173520, 2020, doi: 10.1109/ACCESS.2020.3025032. 
[78] H. Li, F. Fang, Z. Ding, "Joint resource allocation for hybrid NOMA-assisted MEC in 6G networks," in Digital Communications and Networks, vol. 6, no. 3, pp. 241-252, 2020.

[79] H. Xu, P. V. Klaine, O. Onireti, B. Cao, M. Imran, L. Zhang, "Blockchain-enabled resource management and sharing for 6G communications," in Digital Communications and Networks, vol. 6, no. 3, pp. 261-269, 2020.

[80] Y. Wei, M. Peng, Y. Liu, "Intent-based networks for 6G: Insights and challenges," in Digital Communications and Networks, vol. 6, no. 3, pp. 270-280, 2020.

[81] M. Wang, T. Zhu, T. Zhang, J. Zhang, S. Yu, W. Zhou, "Security and privacy in 6G networks: New areas and new challenges," in Digital Communications and Networks, vol. 6, no. 3, pp. 281-291, 2020.

[82] Y. Fu, K. N. Doan, T. Q. S. Quek, “On recommendationaware content caching for 6G: An artificial intelligence and optimization empowered paradigm," in Digital Communications and Networks, vol. 6, no. 3, pp. 304-311, 2020.

[83] Y. Chen, W. Liu, Z. Niu, Z. Feng, Q. Hu, T. Jiang, "Pervasive intelligent endogenous $6 \mathrm{G}$ wireless systems: Prospects, theories and key technologies," in Digital Communications and Networks, vol. 6, no. 3, pp. 312320, 2020.

[84] International Telecommunication Union (ITU), "IMT Vision - Framework and Overall Objectives of the Future Development of IMT for 2020 and Beyond," ITU Publications, M.2083-0, 2015.

[85] International Telecommunication Union (ITU), "Final Acts World Radiocommunication Conference 2019 (WRC-19)," ITU Publications, 2019.

[86] A. Ghosh, A. Maeder, M. Baker and D. Chandramouli, "5G Evolution: A View on 5G Cellular Technology Beyond 3GPP Release 15," in IEEE Access, vol. 7, pp. 127639-127651, 2019, doi: 10.1109/ACCESS.2019.2939938.

[87] P. Kumari, S. A. Vorobyov and R. W. Heath, "Adaptive Virtual Waveform Design for Millimeter-Wave Joint Communication-Radar," in IEEE Transactions on Signal Processing, vol. 68, pp. 715-730, 2020, doi: 10.1109/TSP.2019.2956689.

[88] A. Yazar and H. Arslan, "Flexible Multi-Numerology Systems for 5G New Radio," in River Publishers Journal of Mobile Multimedia, vol. 14 no.4, pp. 367-394, 2018.

[89] A. B. Kihero, M. S. J. Solaija and H. Arslan, "InterNumerology Interference for Beyond 5G," in IEEE Access, vol. 7, pp. 146512-146523, 2019, doi: 10.1109/ACCESS.2019.2946084.
[90] Z. E. Ankarali, B. Peköz and H. Arslan, "Flexible Radio Access Beyond 5G: A Future Projection on Waveform, Numerology, and Frame Design Principles," in IEEE Access, vol. 5, pp. 18295-18309, 2017, doi: 10.1109/ACCESS.2017.2684783.

[91] M. M. Sahin and H. Arslan, "Multi-functional Coexistence of Radar-Sensing and Communication Waveforms," in IEEE Vehicular Technology Conference (VTC-Fall), Victoria, Canada, 2020.

[92] A. A. Sabah and H. Arslan, "NOMA for MultiNumerology OFDM Systems," in Hindawi Wireless Communications and Mobile Computing, vol. 2018, pp. 1-9, 2018, doi:10.1155/2018/8514314.

[93] A. Tusha, S. Doğan and H. Arslan, "A Hybrid Downlink NOMA With OFDM and OFDM-IM for Beyond 5G Wireless Networks," in IEEE Signal Processing Letters, vol. 27, pp. 491-495, 2020, doi: 10.1109/LSP.2020.2979059.

[94] M. M. Şahin and H. Arslan, "Waveform-Domain NOMA: The Future of Multiple Access," 2020 IEEE International Conference on Communications Workshops (ICC Workshops), Dublin, Ireland, 2020, pp. 1-6, doi: 10.1109/ICCWorkshops49005.2020.9145077.

[95] A. Maatouk, E. Çalışkan, M. Koca, M. Assaad, G. Gui and H. Sari, "Frequency-Domain NOMA With Two Sets of Orthogonal Signal Waveforms," in IEEE Communications Letters, vol. 22, no. 5, pp. 906-909, May 2018, doi: 10.1109/LCOMM.2018.2810118.

[96] M. B. Celebi and H. Arslan, "Theoretical Analysis of the Co-Existence of LTE-A Signals and Design of an ML-SIC Receiver," in IEEE Transactions on Wireless Communications, vol. 14, no. 8, pp. 4626-4639, Aug. 2015, doi: 10.1109/TWC.2015.2424244.

[97] A. M. Jaradat, J. M. Hamamreh and H. Arslan, “Modulation Options for OFDM-Based Waveforms: Classification, Comparison, and Future Directions," in IEEE Access, vol. 7, pp. 17263-17278, 2019, doi: 10.1109/ACCESS.2019.2895958.

[98] P. Yang, Y. Xiao, Y. L. Guan, M. Di Renzo, S. Li and L. Hanzo, "Multidomain Index Modulation for Vehicular and Railway Communications: A Survey of Novel Techniques," in IEEE Vehicular Technology Magazine, vol. 13, no. 3, pp. 124-134, Sept. 2018, doi: 10.1109/MVT.2018.2814023.

[99] S. Doğan, A. Tusha and H. Arslan, "NOMA With Index Modulation for Uplink URLLC Through GrantFree Access," in IEEE Journal of Selected Topics in Signal Processing, vol. 13, no. 6, pp. 1249-1257, Oct. 2019, doi: 10.1109/JSTSP.2019.2913981. 
[100] A. Yazar and H. Arslan, "A Flexibility Metric and Optimization Methods for Mixed Numerologies in 5G and Beyond," in IEEE Access, vol. 6, pp. 3755-3764, 2018, doi: 10.1109/ACCESS.2018.2795752.

[101] A. F. Demir and H. Arslan, "Inter-Numerology Interference Management With Adaptive Guards: A CrossLayer Approach," in IEEE Access, vol. 8, pp. 3037830386, 2020, doi: 10.1109/ACCESS.2020.2972287.

[102] E. Memisoglu, A. B. Kihero, E. Basar and H. Arslan, "Guard Band Reduction for $5 \mathrm{G}$ and Beyond Multiple Numerologies," in IEEE Communications Letters, vol. 24, no. 3, pp. 644-647, Mar. 2020, doi: 10.1109/LCOMM.2019.2963311.

[103] A. Yazar and H. Arslan, "Reliability Enhancement in Multi-Numerology Based 5G New Radio Using INIAware Scheduling," in EURASIP Journal on Wireless Communications and Networking, vol. 2019 no. 110, pp. 1-14, 2019.

[104] M. J. Colella, J. N. Martin and F. Akyildiz, "The HALO network ${ }^{\mathrm{TM}}$," in IEEE Communications Magazine, vol. 38, no. 6, pp. 142-148, June 2000, doi: $10.1109 / 35.846086$.

[105] T. Han, X. Ge, L. Wang, K. S. Kwak, Y. Han and X. Liu, "5G Converged Cell-Less Communications in Smart Cities," in IEEE Communications Magazine, vol. 55, no. 3, pp. 44-50, Mar. 2017, doi: 10.1109/MCOM.2017.1600256CM.

[106] L. Wang, T. Han, Q. Li, J. Yan, X. Liu and D. Deng, "Cell-Less Communications in 5G Vehicular Networks Based on Vehicle-Installed Access Points," in IEEE Wireless Communications, vol. 24, no. 6, pp. 64-71, Dec. 2017, doi: 10.1109/MWC.2017.1600401.

[107] M. S. J. Solaija, H. Salman, A. B. Kihero, M. I. Saglam, H. Arslan, "Generalized Coordinated Multipoint Framework for 5G and Beyond," arXiv:2008.06343 [eess.SP], Aug. 2020.

[108] M. S. Ali, E. Hossain, A. Al-Dweik and D. I. Kim, "Downlink Power Allocation for CoMP-NOMA in Multi-Cell Networks," in IEEE Transactions on Communications, vol.66, no. 9, pp. 3982-3998, Sept. 2018, doi: 10.1109/TCOMM.2018.2831206.

[109] J. Ding and J. Cai, "Two-Side Coalitional Matching Approach for Joint MIMO-NOMA Clustering and BS Selection in Multi-Cell MIMO-NOMA Systems," in IEEE Transactions on Wireless Communications, vol. 19, no. 3, pp. 2006-2021, Mar. 2020, doi: 10.1109/TWC.2019.2961654.

[110] B. Han, J. Lianghai and H. D. Schotten, "Slice as an Evolutionary Service: Genetic Optimization for Inter-Slice Resource Management in 5G Networks," in IEEE Access, vol. 6, pp. 33137-33147, 2018, doi: 10.1109/ACCESS.2018.2846543.
[111] D. Sattar and A. Matrawy, "Optimal Slice Allocation in 5G Core Networks," in IEEE Networking Letters, vol. 1, no. 2, pp. 48-51, June 2019, doi: 10.1109/LNET.2019.2908351.

[112] D. A. Chekired, M. A. Togou, L. Khoukhi and A. Ksentini, “5G-Slicing-Enabled Scalable SDN Core Network: Toward an Ultra-Low Latency of Autonomous Driving Service," in IEEE Journal on Selected Areas in Communications, vol. 37, no. 8, pp. 1769-1782, Aug. 2019, doi: 10.1109/JSAC.2019.2927065.

[113] Q. Zhang, H. Sun, Z. Wei and Z. Feng, "Sensing and Communication Integrated System for Autonomous Driving Vehicles," IEEE INFOCOM 2020 - IEEE Conference on Computer Communications Workshops (INFOCOM WKSHPS), Toronto, ON, Canada, 2020 , pp. $1278-1279$, doi: 10.1109/INFOCOMWKSHPS50562.2020.9162963.

[114] H. Turkmen, M. S. J. Solaija, H. M. Furqan, H. Arslan, "Generalized Radio Environment Monitoring for Next Generation Wireless Networks," arXiv:2008.06203 [eess.SP], Aug. 2020.

[115] W. Lee and I. F. Akyildiz, "Optimal spectrum sensing framework for cognitive radio networks," in IEEE Transactions on Wireless Communications, vol. 7, no. 10 , pp. $3845-3857$, October 2008, doi: 10.1109/TWC.2008.070391.

[116] S. Yarkan and H. Arslan, "Exploiting location awareness toward improved wireless system design in cognitive radio," in IEEE Communications Magazine, vol. 46, no. 1, pp. 128-136, Jan. 2008, doi: 10.1109/MCOM.2008.4427241.

[117] H. F. Ates, S. M. Hashir, T. Baykas and B. K. Gunturk, "Path Loss Exponent and Shadowing Factor Prediction From Satellite Images Using Deep Learning," in IEEE Access, vol. 7, pp. 101366-101375, 2019, doi: 10.1109/ACCESS.2019.2931072.

[118] D. Sabella et al., "A flexible and reconfigurable 5G networking architecture based on context and content information," 2017 European Conference on Networks and Communications (EuCNC), Oulu, 2017, pp. 1-6, doi: 10.1109/EuCNC.2017.7980669.

[119] Z. Feng, Z. Fang, Z. Wei, X. Chen, Z. Quan and D. Ji, "Joint radar and communication: A survey," in China Communications, vol. 17 , no. 1, pp. 1-27, Jan. 2020, doi: 10.23919/JCC.2020.01.001.

[120] G. Naik, J. Park, J. Ashdown, W. Lehr, "Next Generation Wi-Fi and 5G NR-U in the $6 \mathrm{GHz}$ Bands: Opportunities \& Challenges," arXiv:2006.16534, 2020.

[121] H. B. Yilmaz, T. Tugcu, F. Alagöz and S. Bayhan, "Radio environment map as enabler for practical cognitive radio networks," in IEEE Communications Magazine, vol. 51, no. 12, pp. 162-169, Dec. 2013, doi: 10.1109/MCOM.2013.6685772. 
[122] A. Imran, A. Zoha and A. Abu-Dayya, "Challenges in $5 G$ : how to empower SON with big data for enabling 5G," in IEEE Network, vol. 28, no. 6, pp. 27-33, Nov.Dec. 2014, doi: 10.1109/MNET.2014.6963801.

[123] Q. Mao, F. Hu and Q. Hao, "Deep Learning for Intelligent Wireless Networks: A Comprehensive Survey," in IEEE Communications Surveys \& Tutorials, vol. 20, no. 4, pp. 2595-2621, Fourthquarter 2018, doi: 10.1109/COMST.2018.2846401.

[124] C. Zhang, P. Patras and H. Haddadi, "Deep Learning in Mobile and Wireless Networking: A Survey," in IEEE Communications Surveys \& Tutorials, vol. 21, no. 3, pp. 2224-2287, thirdquarter 2019, doi: 10.1109/COMST.2019.2904897.

[125] N. C. Luong et al., "Applications of Deep Reinforcement Learning in Communications and Networking: A Survey," in IEEE Communications Surveys \& Tutorials, vol. 21, no. 4, pp. 3133-3174, Fourthquarter 2019, doi: 10.1109/COMST.2019.2916583.

[126] Y. Sun, M. Peng, Y. Zhou, Y. Huang and S. Mao, "Application of Machine Learning in Wireless Networks: Key Techniques and Open Issues," in IEEE Communications Surveys \& Tutorials, vol. 21, no. 4, pp. 3072-3108, Fourthquarter 2019, doi: 10.1109/COMST.2019.2924243.

[127] M. Chen, U. Challita, W. Saad, C. Yin and M. Debbah, "Artificial Neural Networks-Based Machine Learning for Wireless Networks: A Tutorial," in IEEE Communications Surveys \& Tutorials, vol. 21, no. 4, pp. 3039-3071, Fourthquarter 2019, doi: 10.1109/COMST.2019.2926625.

[128] J. Wang, C. Jiang, H. Zhang, Y. Ren, K. Chen and L. Hanzo, "Thirty Years of Machine Learning: The Road to Pareto-Optimal Wireless Networks," in IEEE Communications Surveys \& Tutorials, doi: 10.1109/COMST.2020.2965856.

[129] X. Wang, Y. Han, V. C. M. Leung, D. Niyato, X. Yan and X. Chen, "Convergence of Edge Computing and Deep Learning: A Comprehensive Survey," in IEEE Communications Surveys \& Tutorials, vol. 22 , no. 2, pp. 869-904, Secondquarter 2020, doi: 10.1109/COMST.2020.2970550.

[130] C. Jiang, H. Zhang, Y. Ren, Z. Han, K. Chen and L. Hanzo, "Machine Learning Paradigms for NextGeneration Wireless Networks," in IEEE Wireless Communications, vol. 24, no. 2, pp. 98-105, Apr. 2017, doi: 10.1109/MWC.2016.1500356WC.

[131] R. Li et al., "Intelligent 5G: When Cellular Networks Meet Artificial Intelligence," in IEEE Wireless Communications, vol. 24, no. 5, pp. 175-183, Oct. 2017, doi: 10.1109/MWC.2017.1600304WC.
[132] Z. Chang, L. Lei, Z. Zhou, S. Mao and T. Ristaniemi, "Learn to Cache: Machine Learning for Network Edge Caching in the Big Data Era," in IEEE Wireless Communications, vol. 25, no. 3, pp. 28-35, June 2018, doi: 10.1109/MWC.2018.1700317.

[133] E. Basar, M. Di Renzo, J. De Rosny, M. Debbah, M. Alouini and R. Zhang, "Wireless Communications Through Reconfigurable Intelligent Surfaces," in IEEE Access, vol. 7, pp. 116753-116773, 2019, doi: 10.1109/ACCESS.2019.2935192.

[134] M. D. Renzo et al., "Smart Radio Environments Empowered by Reconfigurable Intelligent Surfaces: How it Works, State of Research, and Road Ahead," in IEEE Journal on Selected Areas in Communications, doi: 10.1109/JSAC.2020.3007211.

[135] C. Xu, L. Yang and P. Zhang, "Practical Backscatter Communication Systems for Battery-Free Internet of Things: A Tutorial and Survey of Recent Research," in IEEE Signal Processing Magazine, vol. 35, no. 5, pp. 16-27, Sept. 2018, doi: 10.1109/MSP.2018.2848361.

[136] N. Van Huynh, D. T. Hoang, X. Lu, D. Niyato, P. Wang and D. I. Kim, "Ambient Backscatter Communications: A Contemporary Survey," in IEEE Communications Surveys \& Tutorials, vol. 20, no. 4, pp. 2889-2922, Fourthquarter 2018, doi: 10.1109/COMST.2018.2841964.

[137] R. Long, Y. Liang, H. Guo, G. Yang and R. Zhang, "Symbiotic Radio: A New Communication Paradigm for Passive Internet of Things," in IEEE Internet of Things Journal, vol. 7, no. 2, pp. 1350-1363, Feb. 2020, doi: 10.1109/JIOT.2019.2954678.

[138] Q. Zhang, Y. Liang and H. V. Poor, "Symbiotic Radio: A New Application of Large Intelligent Surface/Antennas (LISA)," 2020 IEEE Wireless Communications and Networking Conference (WCNC), Seoul, Korea (South), 2020, pp. 1-6, doi: 10.1109/WCNC45663.2020.9120455.

[139] S. Buzzi, C. I, T. E. Klein, H. V. Poor, C. Yang and A. Zappone, "A Survey of Energy-Efficient Techniques for $5 G$ Networks and Challenges Ahead," in IEEE Journal on Selected Areas in Communications, vol. 34, no. 4, pp. 697-709, Apr. 2016, doi: 10.1109/JSAC.2016.2550338.

[140] J. Huang, C. Xing and C. Wang, "Simultaneous Wireless Information and Power Transfer: Technologies, Applications, and Research Challenges," in IEEE Communications Magazine, vol. 55, no. 11, pp. 26-32, Nov. 2017, doi: 10.1109/MCOM.2017.1600806.

[141] T. D. Ponnimbaduge Perera, D. N. K. Jayakody, S. K. Sharma, S. Chatzinotas and J. Li, "Simultaneous Wireless Information and Power Transfer (SWIPT): Recent Advances and Future Challenges," 
in IEEE Communications Surveys \& Tutorials, vol. 20, no. 1, pp. 264-302, Firstquarter 2018, doi: 10.1109/COMST.2017.2783901.

[142] J. M. Hamamreh, H. M. Furqan and H. Arslan, “Classifications and Applications of Physical Layer Security Techniques for Confidentiality: A Comprehensive Survey," in IEEE Communications Surveys \& Tutorials, vol. 21, no. 2, pp. 1773-1828, Secondquarter 2019, doi: 10.1109/COMST.2018.2878035.

[143] J. M. Hamamreh, M. Yusuf, T. Baykas and $H$. Arslan, "Cross MAC/PHY layer security design using ARQ with MRC and adaptive modulation," 2016 IEEE Wireless Communications and Networking Conference, Doha, 2016, pp. 1-7, doi: 10.1109/WCNC.2016.7564987.

[144] S. Dwivedi, M. Zoli, A. N. Barreto, P. Sen and G. Fettweis, "Secure Joint Communications and Sensing using Chirp Modulation," 2020 2nd 6G Wireless Summit (6G SUMMIT), Levi, Finland, 2020, pp. 1-5, doi: 10.1109/6GSUMMIT49458.2020.9083884.

[145] Y. Hu and R. Zhang, "A Spatiotemporal Approach for Secure Crowdsourced Radio Environment Map Construction," in IEEE/ACM Transactions on Networking, doi: 10.1109/TNET.2020.2992939.

\section{AUTHORS}

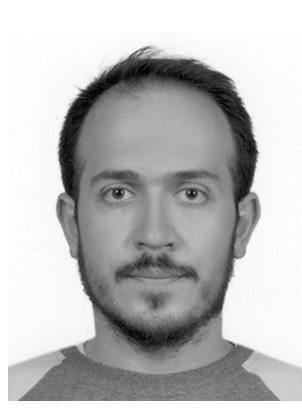

A. Yazar received his B.Sc. degree in electrical engineering from Eskisehir Osmangazi University, Eskisehir, Turkey in 2011, M.Sc. degree in electrical engineering from Bilkent University, Ankara, Turkey in 2013, and Ph.D. degree in electrical engineering from Istanbul Medipol University, Istanbul, Turkey in 2020. He is currently general coordinator as a member of the Communications, Signal Processing, and Networking Center (CoSiNC) at Istanbul Medipol University. His current research interests are flexible waveform design, radio resource management techniques, and the role of machine learning in wireless communications systems.

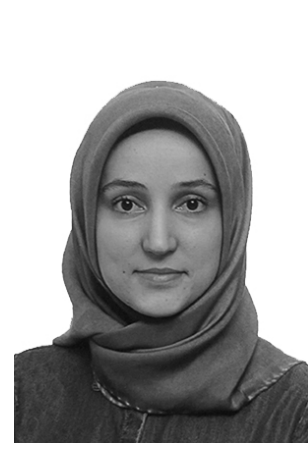

S. Doğan Tusha received the B.Sc. degree in electronics and telecommunication engineering from Kocaeli University, Kocaeli, Turkey, in 2015, and the Ph.D. degree in electrical and electronics engineering from Istanbul Medipol University, Istanbul, Turkey, in 2020. She is currently a post-doctoral researcher in the Communications, Signal Processing, and Networking Center (CoSiNC) at Istanbul Medipol University, Istanbul, Turkey. Her research interests include index modulation, millimeter-wave frequency bands, nonorthogonal multiple accessing (NOMA), and random access techniques for next-generation wireless networks.

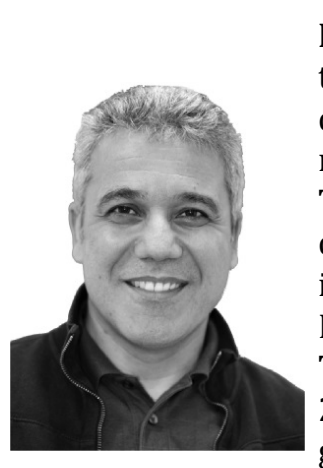

H. Arslan (IEEE Fellow, IEEE Distinguished Lecturer) received his BS degree from the Middle East Technical University (METU), Ankara, Turkey in 1992; his MS and Ph.D. degrees were received respectively in 1994 and 1998 from Southern Methodist University (SMU), Dallas, TX. From January 1998 to August 2002, he was with the research group of Ericsson, where he was involved with several projects related to $2 \mathrm{G}$ and $3 \mathrm{G}$ wireless communication systems. Since August 2002, he has been with the Electrical Engineering Department, at the University of South Florida, where he is a Professor. In December 2013, he joined Istanbul Medipol University to found the Engineering College, where he has worked as the Dean of the School of Engineering and Natural Sciences. In addition, he has worked as a part-time consultant for various companies and institutions including Anritsu Company and The Scientific and Technological Research Council of Turkey. 
Dr. Arslan conducts research in wireless systems, with emphasis on the physical and medium access layers of communications. His current research interests are on 5G and beyond radio access technologies, physical layer security, interference management (avoidance, awareness, and cancellation), cognitive radio, multi-carrier wireless technologies (beyond OFDM), dynamic spectrum access, coexistence issues, non-terrestial communications (High Altitude Platforms), joint radar (sensing) and communication designs. Dr. Arslan has been collaborating extensively with key national and international industrial partners and his research has generated significant interest in companies such as InterDigital, Anritsu, NTT DoCoMo, Raytheon, Honeywell, Keysight technologies. Collaborations and feedback from industry partners has significantly influenced his research. In addition to his research activities, Dr. Arslan has also contributed to wireless communication education. He has integrated the outcomes of his research into education which lead him to develop a number of courses at the University of South Florida. He has developed a unique "Wireless Systems Laboratory" course (funded by the National Science Foundation and Keysight technologies) where he was able to teach not only the theory but also the practical aspects of wireless communication system with the most contemporary test and measurement equipment.

Dr. Arslan has served as general chair, technical program committee chair, session and symposium organizer, workshop chair, and technical program committee member in several IEEE conferences. He is currently a member of the editorial board for the IEEE Surveys and Tutorials and the Sensors Journal. He has also served as a member of the editorial board for the IEEE Transactions on Communications, the IEEE Transactions on Cognitive Communications and Networking (TCCN), and several other scholarly journals by Elsevier, Hindawi, and Wiley Publishing. 